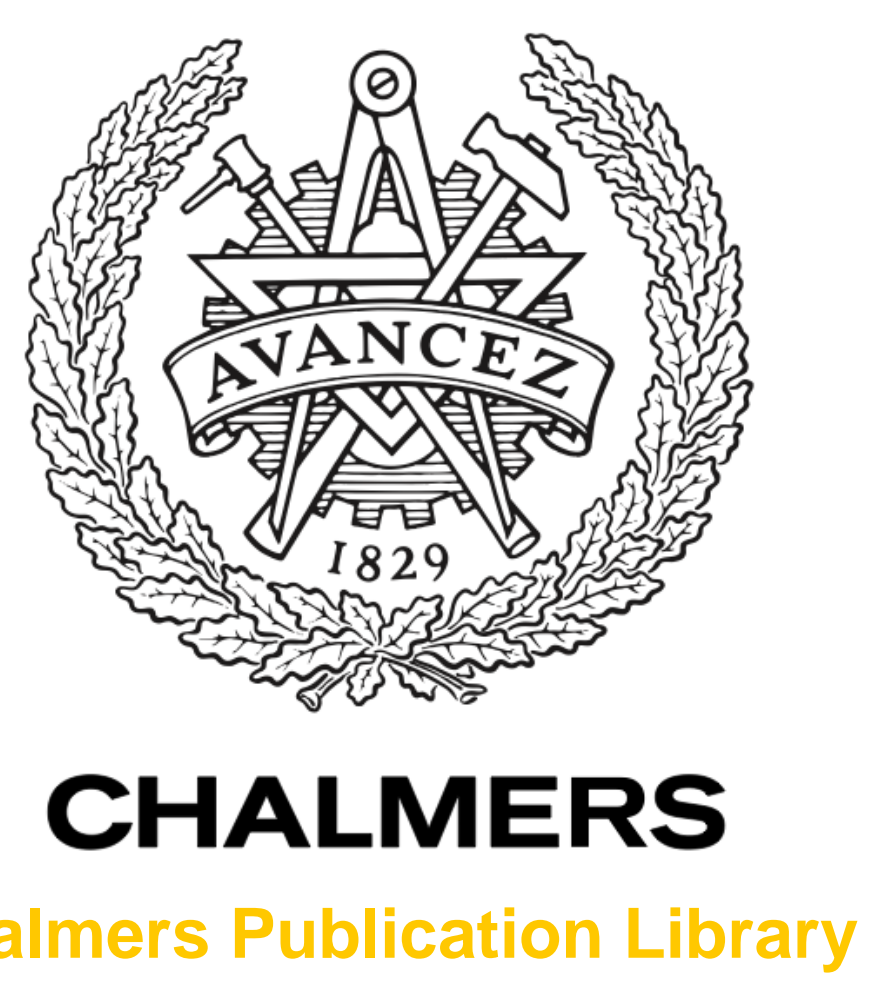

Chalmers Publication Library

\title{
Cluster-Based Radio Resource Management for D2D-Supported Safety-Critical V2X Communications
}

This document has been downloaded from Chalmers Publication Library $(\mathrm{CPL})$. It is the author's version of a work that was accepted for publication in:

IEEE Transactions on Wireless Communications (ISSN: 1536-1276)

Citation for the published paper:

Wanlu, S. ; Yuan, D. ; Ström, E. et al. (2016) "Cluster-Based Radio Resource Management for D2D-Supported Safety-Critical V2X Communications". IEEE Transactions on Wireless Communications

Downloaded from: http://publications.lib.chalmers.se/publication/231485

Notice: Changes introduced as a result of publishing processes such as copy-editing and formatting may not be reflected in this document. For a definitive version of this work, please refer to the published source. Please note that access to the published version might require a subscription. 


\title{
Cluster-Based Radio Resource Management for D2D-Supported Safety-Critical V2X Communications
}

\author{
Wanlu Sun, Di Yuan, Senior Member, IEEE, Erik G. Ström, Senior Member, IEEE, \\ and Fredrik Brännström, Member, IEEE
}

\begin{abstract}
Deploying direct device-to-device (D2D) links is a promising technology for vehicle-to-X (V2X) applications. However, intra-cell interference, along with stringent requirements on latency and reliability, are challenging issues. In this paper, we study the radio resource management problem for D2Dbased safety-critical V2X communications. We first transform the $\mathrm{V} 2 \mathrm{X}$ requirements into the constraints that are computable using slowly varying channel state information only. Secondly, we formulate an optimization problem, taking into account the requirements of both vehicular users (V-UEs) and cellular users (C-UEs), where resource sharing can take place not only between a V-UE and a C-UE but also among different VUEs. The NP-hardness of the problem is rigorously proved. Moreover, a heuristic algorithm, called Cluster-based Resource block sharing and pOWer allocatioN (CROWN), is proposed to solve this problem. Finally, simulations results indicate promising performance of the CROWN scheme.
\end{abstract}

\section{INTRODUCTION}

\section{A. Motivation}

Recently, vehicles became the third fastest growing type of connected devices after smart phones and tablets [1]. As a result, communication in moving networks is attracting great interests. In this context, V2X communication plays a crucial role since it enables reliable and low-latency services such as traffic safety systems [2]. Here "V2X" is a term that collectively refers to vehicle-to-vehicle (V2V), vehicleto-infrastructure (V2I), and vehicle-to-device (V2D). In this work, we focus on safety-critical V2X applications, which usually have a strongly localized nature and finite transmit buffers. Besides, V2X applications have to be real time and come with stringent requirement on reliability. Here we assume hard deadlines for the applications, i.e., the transmitted message is considered useless when its latency exceeds the deadline and there is no additional benefit if the latency is less than the deadline. Note that this is in contrast with soft deadlines, i.e., the value of the message decreases smoothly with the latency. For instance, the European Union METIS

Wanlu Sun, Erik G. Ström, and Fredrik Brännström are with the Division of Communication Systems, Department of Signals and Systems, Chalmers University of Technology, SE-412 96 Gothenburg, Sweden. E-mail: \{wanlu, erik.strom, fredrik.brannstrom\}@ chalmers.se

Department of Science and Technology, Linköping University, 60174 Norrköping, Sweden. E-mail: di.yuan@liu.se

This work has been supported in part by the Swedish Research Council project 2011-5824. Part of this work has been performed in the framework of the FP7 project ICT-317669 METIS, which is partly funded by the EU. The authors would like to acknowledge the contributions of their colleagues in METIS, although the views expressed are those of the authors and do not necessarily represent the project. The work of the second author has been supported by European FP7 Marie Curie IOF Grant 329313. The calculations were performed on resources provided by the Swedish National Infrastructure for Computing (SNIC) at C3SE. project [2] considers a maximum end-to-end delay of $5 \mathrm{~ms}$ and transmission reliability of $99.999 \%$.

Current state of the art solutions for V2X are ad-hoc communications based on the IEEE 802.11p standard and backend-based communications over the Long Term Evolution (LTE) cellular standard. However, as analyzed in [3], [4], these two solutions do not fullfill the stringent quality of service (QoS) requirements of the most demanding V2X applications.

A related trend is the emergence of device-to-device (D2D) communication, which has been identified as one of the technology components for future cellular systems [2], [5]. In a D2D underlaying cellular infrastructure, two physically close user equipment (UE) devices can directly communicate with each other by sharing the same resources used by regular cellular UEs (C-UEs), with the benefits of proximity gain, reuse gain, and hop gain [6]. In fact, the direct D2D link is a promising enabler for safety-critical V2X communication. Firstly, the localized nature of V2X services is exactly the motivating idea for D2D communication. Moreover, the low latency requirement of $\mathrm{V} 2 \mathrm{X}$ applications matches well the hop gain of D2D transmission. Last but not least, the requirement for $\mathrm{V} 2 \mathrm{X}$ communication on high reliability is congruent with the proximity gain provided by D2D links. However, using D2D underlay for V2X communication leads to interference from resource reuse. Also, guaranteeing the required latency and reliability for $\mathrm{V} 2 \mathrm{X}$ services is challenging. Hence, radio resource management (RRM) becomes a key design aspect to enable D2D-supported V2X communications.

\section{B. State of the Art}

RRM strategies for conventional D2D systems have been extensively researched in [6]-[12], to name a few. Studied issues include how C-UEs and D2D UEs share resource blocks (RBs) and how each UE allocates its transmit power among the RBs. For more details on this line of research, readers are referred to the surveys in [6]-[8] and the references therein. However, we note that there are three major limitations in most of the existing D2D work for our target application of safety-critical V2X.

Firstly, the performance objective has typically been to maximize the sum rate and prioritize cellular links [11][13]. Thus the D2D underlay is considered opportunistic as their interference to cellular links is controlled to be at acceptable levels. As a result, schemes for traditional D2D systems do not work well for V2X applications that have small message payloads and very strict requirements on latency and reliability.

Secondly, the majority of the literature assumes that the eNB is aware of the complete instantaneous channel state informa- 
tion (CSI) of all the cellular and D2D links; this assumption is too optimistic for highly dynamic moving networks. A more practical solution is to consider knowledge of partial CSI consisting only of slowly varying parameters (path loss and shadowing). This, however, gives rise to another challenge: how to transform the requirements of V2X communications, which are subject to random fast fading effects, into constraints that only require slowly varying CSI. Efforts along this direction can be found in [14], [15], which, however, have some limitations that will be detailed in Section III-A.

Thirdly, most previous studies consider the setup when an RB can be shared by at most one D2D link [4], [11], [12], i.e., orthogonal RB allocation among D2D UEs. In fact, as long as link quality can be assured, allowing multiple and concurrent D2D transmissions on the same RB (i.e., non-orthogonal access for D2D UEs) will not only improve spectrum efficiency, but may also lead to less interference to C-UEs due to spatial reuse. Recently, the authors of [7], [13], [16] allowed multiple D2D links to share the same RB; the setup in [7], [13], [16], however, does not apply D2D to V2X communications.

When it comes to using a D2D underlay for V2X communication, to the best of our knowledge, only a few studies [1]-[4], [17], [18] have been conducted. The suitability of the D2D technique to V2X applications was systematically discussed in [1], [2], [18]. The authors of [3] proposed a heuristic location-dependent resource allocation scheme to protect the communication of vehicular UEs (V-UEs). The performance of C-UEs, however, was not optimized. In [4], considering QoS requirements of both V-UEs and C-UEs, we proposed an RB allocation and power control (SRBP) scheme for D2Dbased $\mathrm{V} 2 \mathrm{~V}$ communications, where RB allocation is restricted to be orthogonal among the V-UEs. Furthermore, allowing non-orthogonal access for V-UEs, we proposed a two-stage RB sharing and power allocation (RBSPA) algorithm in [17], where Perron-Frobenius theory was utilized to design an RB sharing metric.

\section{Contributions}

In this work, we study the RRM problem for D2D-supported safety-critical V2X communication. The main contributions are as follows.

- We propose an improved analytical method compared to [17] to transform the strict latency and reliability requirements of V2X communication, which are subject to random fast fading effects, into optimization constraints that are computable with slowly varying CSI only. This transformation allows for extending some existing D2D RRM algorithms, e.g., [11], [12], to cater also for V2V communications.

- Allowing non-orthogonal access for V-UEs, we formulate an RRM problem of RB sharing and power allocation for a set of C-UEs and V-UEs as in [17]. This is stated as an optimization problem with the objective of maximizing the C-UE sum rate with proportional bandwidth fairness, under the constraint of satisfying the V-UEs requirements on latency and reliability. Moreover, we provide a math- ematical proof of the problem's NP-hardness, which is absent in [17].

- Due to the NP-hardness, we propose a novel three-stage heuristic RRM solution, referred to as the Cluster-based RB sharing and pOWer allocatioN (CROWN) algorithm, to solve the problem; the algorithm requires only slowly varying CSI at the eNB. Unlike the RBSPA scheme presented in [17] which uses Perron-Frobenius theory, CROWN is designed based on matching theory. Besides, we provide simulations to evaluate the proposed CROWN algorithm and compare its performance with some existing RRM methods, e.g., the RBSPA in [17]. The results illustrate promising performance of the CROWN scheme and its simplified version called CROWN-noPA, in which the last power allocation stage is removed.

\section{Preliminaries}

\section{A. Notation}

We use the following notation throughout the paper. Sets are denoted by calligraphic letters, e.g., $\mathcal{X}$, with $|\mathcal{X}|$ denoting its cardinality. Lowercase and uppercase letters, e.g., $x$ and $X$, represent scalars, lowercase boldface letters, e.g., $\boldsymbol{x}$, designate column vectors where $x_{i}$ indicates the $i$ th element, and uppercase boldface letters, e.g., $\boldsymbol{X}$, denote matrices where $X_{i, j}$ denotes the $(i, j)$ th element. The superscript $(\cdot)^{\top}$ stands for the transposition, and $\mathbf{1}$ and $\mathbf{0}$ represent the all-ones column vector and the zero column vector, respectively. Unless otherwise specified, vector and matrix inequalities are interpreted element-wise. $\mathbb{E}[\cdot]$ indicates the expectation. Key mathematical notation is summarized in Table I.

\section{B. System Model}

Consider a single cell environment with $M^{\prime}$ C-UEs and $K^{\prime}$ V-UEs. The latter is counted in terms of transmitters. The corresponding sets are denoted by $\mathcal{M}^{\prime} \triangleq\left\{1,2, \ldots, M^{\prime}\right\}$ and $\mathcal{K}^{\prime} \triangleq\left\{1,2, \ldots, K^{\prime}\right\}$, respectively. The D2D underlay is only used by V-UEs that share the uplink radio resources. The uplink bandwidth is divided into $F$ RBs for each scheduling time unit. The C-UE RB allocation is assumed to be orthogonal and can be performed by any reasonable scheduling scheme. On the other hand, and unlike existing works [4], [12], a certain CUE's RBs may be shared with multiple V-UEs simultaneously. Hence, the V-UEs RB allocation is, in general, nonorthogonal [17]. Moreover, one V-UE may reuse the RBs of multiple CUEs. Clearly, intra-cell interference may arise in this setup.

Fig. 1 illustrates the communication scenario when C-UE $m^{\prime}$, V-UE pairs $k^{\prime}$ and $l^{\prime}$ are using the same RB. In general, we denote the average power gain for desired channels by $h$ and for interference channels by $g$. Moreover, primed channels, i.e., $h^{\prime}$ or $g^{\prime}$, refers to channels from a C-UE. Primed indicies, e.g., $k^{\prime}$ and $m^{\prime}$, refers to real C-UEs and V-UEs and unprimed indicies, e.g., $k$ and $m$, indicate sub-UEs. The notion of subUEs will be properly defined in Section IV. Hence, in Fig. 1, $h_{m^{\prime}}^{\prime}, h_{k^{\prime}}$, and $h_{l^{\prime}}$ are the average channel power gains of the three desired transmissions, respectively, $g_{m^{\prime}, k^{\prime}}^{\prime}$ and $g_{m^{\prime}, l^{\prime}}^{\prime}$ denote the two interference channel average power gains from the C-UE to the receivers of V-UE $k^{\prime}$ and $l^{\prime}$, respectively, and 
Table I

Key Mathematical Symbols

\begin{tabular}{|c|c|}
\hline Symbol & Definition \\
\hline$C$ & Number of V-UE clusters \\
\hline$E_{k^{\prime}}^{\text {all }}$ & Total number of RBs allocated for the transmission of V-UE $k^{\prime}$ \\
\hline$E_{k^{\prime}}$ & Number of RBs for V-UE $k^{\prime}$ per scheduling time unit \\
\hline$E_{m^{\prime}}^{\prime}$ & Number of RBs for C-UE $m^{\prime}$ per scheduling time unit \\
\hline$F$ & Number of RBs per scheduling time unit \\
\hline$g_{k^{\prime}}$ & Average power gain of interference channel from V-UE $k^{\prime}$ Tx to the eNB \\
\hline$g_{k^{\prime}, l^{\prime}}$ & Average power gain of interference channel from V-UE $k^{\prime}$ Tx to V-UE $l^{\prime}$ Rx \\
\hline$g_{m^{\prime}, k^{\prime}}^{\prime}$ & Average power gain of interference channel from C-UE $m^{\prime}$ to V-UE $k^{\prime} \mathrm{Rx}$ \\
\hline$h_{k^{\prime}}$ & Average power gain of desired channel for V-UE pair $k^{\prime}$ \\
\hline$h_{m^{\prime}}^{\prime}$ & Average power gain of desired channel from C-UE $m^{\prime}$ to the eNB \\
\hline$\hat{k}(k)$ & The mapping from sub-V-UE $k$ to the V-UE that $k$ belongs to \\
\hline$K$ & Number of sub-V-UEs \\
\hline$K^{\prime}$ & Number of V-UEs \\
\hline $\mathcal{K}$ & Set of sub-V-UEs $\{1,2, \ldots, K\}$ \\
\hline $\mathcal{K}^{\prime}$ & Set of V-UEs $\left\{1,2, \ldots, K^{\prime}\right\}$ \\
\hline $\mathcal{K}^{(c)}$ & Set of sub-V-UEs in cluster $c$ \\
\hline $\mathcal{K}^{\prime(c)}$ & Set of V-UEs in cluster $c$ \\
\hline $\mathcal{K}^{(1: c)}$ & Set of sub-V-UEs in clusters $1, \ldots, c$, i.e., $\mathcal{K}^{(1: c)} \triangleq \mathcal{K}^{(1)} \cup \cdots \cup \mathcal{K}^{(c)}$ \\
\hline$\hat{m}(m)$ & The mapping from sub-C-UE $m$ to the C-UE that $m$ belongs to \\
\hline$M$ & Number of sub-C-UEs \\
\hline $\bar{M}$ & Number of C-UEs \\
\hline $\mathcal{M}$ & Set of sub-C-UEs $\{1,2, \ldots, M\}$ \\
\hline $\mathcal{M}^{\prime}$ & Set of C-UEs $\left\{1,2, \ldots, M^{\prime}\right\}$ \\
\hline$P_{m, k}$ & Transmit power of sub-V-UE $k$ on the RB used by sub-C-UE $m$ \\
\hline$S_{m}$ & Transmit power of sub-C-UE $m$ \\
\hline$x_{m, k}$ & Indicator of RB sharing between sub-C-UE $m$ and sub-V-UE $k$ \\
\hline
\end{tabular}

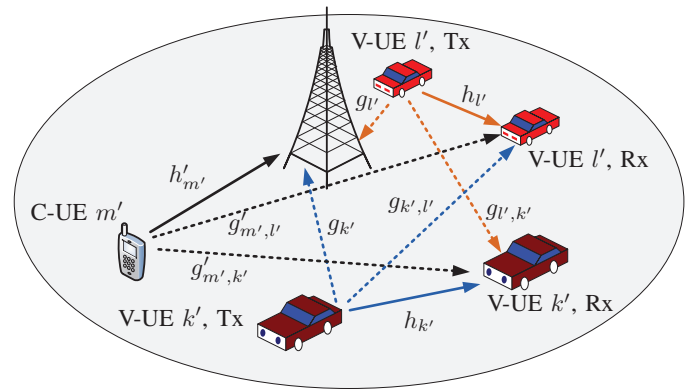

Figure 1. Illustration of $\mathrm{V} 2 \mathrm{X}$ and cellular communications with channel average power gains.

$g_{k^{\prime}}$ and $g_{l^{\prime}}$ are the interfering channel average power gains of the two V-UE transmissions to the eNB. Moreover, $g_{k^{\prime}, l^{\prime}}$ and $g_{l^{\prime}, k^{\prime}}$ represent the cross-talk, interference channel average power gains between the two V-UEs. We define $g_{k^{\prime}, k^{\prime}} \triangleq 0$ for all $k^{\prime} \in \mathcal{K}^{\prime}$. See also Table I.

To perform RRM, the eNB needs some degree of knowledge of the CSI for all involved links. The average power gains $h_{m^{\prime}}^{\prime}$, $g_{k^{\prime}}$, and $g_{l^{\prime}}$ can be measured at the eNB itself, but all other links have to be measured by the corresponding V-UE receiver and then reported to the eNB. The channel power gains are measured over a relative long time period such that the small scale fading effects are averaged out when there is mobility. We note, however, that for a network without mobility, the small scale fading does not vary in time, and the measured channel gains therefore coincide with the instantaneous CSI.

\section{Time Scale and Channel Acquisition for RRM}

One potential advantage of D2D communication is to offload the eNB scheduler [6]. To achieve this offloading gain in reality, the time scale of interactions between the eNB and D2D UEs should be much longer than the traditional LTE scheduling time interval $(1 \mathrm{~ms})$. Furthermore, due to mobility, the channels related to V-UEs are typically highly dynamic. In this case, short-term RRM at the eNB based on instantaneous CSI requires a large overhead since the V-UEs need to report their channel measurements every millisecond or so. For these two reasons, we argue that the eNB should perform long-term RRM, i.e., RRM based on only slowly varying CSI (i.e., path loss and shadowing). Even at very high vehicular speeds, the slowly varying CSI can be considered to be approximately constant for a few hundred milliseconds. From now on, we assume that the slowly varying CSI is known at the eNB. This can be achieved if the V-UEs report averaged CSI measurements to the eNB every few hundred milliseconds. Since path loss and shadowing are not greatly influenced by frequency in the considered bandwidth, the available CSI is considered frequency-invariant. Nevertheless, in Section VI, we will also consider fast fading in simulations to evaluate the validity of the proposed RRM scheme under random channel conditions.

\section{REQUiREMENTS ON V-UES AND C-UES}

Naturally, the QoS requirements of V-UEs and C-UEs are different. In this section, we mathematically formulate the requirements for both of them. 


\section{A. Requirements of $V$-UEs}

For safety-critical V2X services, there are stringent latency and reliability requirements, though high data rate is of less significance. Hence, it is natural to consider the V-UE QoS requirements as constraints in a mathematical optimization problem.

Assume that $E_{k^{\prime}}^{\text {all }} \mathrm{RBs}$ are allocated to V-UE $k^{\prime}$. According to [19], the outage probability, i.e., the probability that $N_{k^{\prime}}$ error-free bits cannot be delivered by any coding scheme, is equal to

$$
p_{k^{\prime}}^{\text {out }} \triangleq \operatorname{Pr}\left\{\sum_{i=1}^{\left.E_{k^{\prime}}^{\text {all }} \rho \log _{2}\left(1+\gamma_{i}\right)<N_{k^{\prime}}\right\}}\right.
$$

where $\gamma_{i} \triangleq \bar{P}_{i}^{\mathrm{r}}\left|H_{i}\right|^{2} /\left(\sigma^{2}+\sum_{j \neq i} \bar{S}_{j, i}^{\mathrm{r}}\left|G_{j, i}\right|^{2}\right)$ is the instantaneous Signal to Interference plus Noise Ratio (SINR) of RB $i$; $\bar{P}_{i}^{\mathrm{r}}$ and $\bar{S}_{j, i}^{\mathrm{r}}$ are average received power from the desired and interfering users, respectively; $H_{i}$ and $G_{j, i}$ are independent random variables with $\mathbb{E}\left[\left|H_{i}\right|^{2}\right]=1$ and $\mathbb{E}\left[\left|G_{j, i}\right|^{2}\right]=1$ that model the fast fading effects of the corresponding desired channel and interference channel; and $\sigma^{2}$ is the noise power. The multiplication with $\rho$ is due to the fact that an RB contains $\rho$ complex symbols. Then, the reliability requirement is interpreted from the perspective of outage probability and can be expressed as [2]

$$
p_{k^{\prime}}^{\text {out }} \leq p_{\mathrm{o}}
$$

where $p_{\mathrm{o}}$ is the maximum tolerable outage probability. Note that in our problem, the outage probability is measured in terms of the aggregated number of bits that can be transmitted (i.e., the summation in (1)) rather than in terms of the SINR. For safety-critical V2X communications, the former is more reasonable since the requirements are usually expressed as that a certain amount of data needs to be delivered within a certain time period with a given probability [2], [20], [21].

However, since only slowly varying CSI is available at the eNB, it is necessary to devise constraints that (a) involve only slowly varying CSI and (b) imply that (2) is satisfied. For this line of research, the authors of [14], [15] derived mappings to relate the outage probability to an average SINR margin, when outage is based on the instantaneous SINR. However, how to derive the relationship between the outage probability in (1) and an average SINR margin remains an open issue. To address this problem, we will replace the requirement in (2) by a more strict requirement shown in Lemma 1 .

Lemma 1. For all $i, j$, suppose that $\left|H_{i}\right|^{2}$ and $\left|G_{j, i}\right|^{2}$ are statistically independent unit mean random variables. Moreover, suppose that the cumulative distribution function (CDF) of each $\left|H_{i}\right|^{2}$ is concave on $\mathbb{R}_{+}$. Let

$$
\bar{\gamma}_{k^{\prime}}^{\mathrm{T}} \triangleq \arg \min _{\gamma \in \mathcal{R}_{+}} \operatorname{Pr}\left\{\sum_{i=1}^{E_{k^{\prime}}^{\mathrm{all}}} \rho \log _{2}\left(1+\gamma\left|H_{i}\right|^{2}\right)<N_{k^{\prime}}\right\} \leq p_{\mathrm{o}}
$$

where $\gamma$ is the optimization variable of the minimization problem in (3). Let

$$
\bar{\gamma}_{i} \triangleq \frac{\bar{P}_{i}^{\mathrm{r}}}{\sigma^{2}+\sum_{j \neq i} \bar{S}_{j, i}^{\mathrm{r}}},
$$

where $\bar{P}_{i}^{\mathrm{r}}, \bar{S}_{j, i}^{\mathrm{r}}$, and $\sigma^{2}$ are non-negative constants, and consider

$$
\bar{\gamma}_{i} \geq \bar{\gamma}_{k^{\prime}}^{\mathrm{T}}, \quad \text { for all } i=1,2, \ldots, E_{k^{\prime}}^{\text {all }}
$$

If (5) is satisfied, then

$\operatorname{Pr}\left\{\sum_{i=1}^{E_{k^{\prime}}^{\text {all }}} \rho \log _{2}\left(1+\frac{\bar{P}_{i}^{\mathrm{r}}\left|H_{i}\right|^{2}}{\sigma^{2}+\sum_{j \neq i} \bar{S}_{j, i}^{\mathrm{r}}\left|G_{j, i}\right|^{2}}\right)<N_{k^{\prime}}\right\} \leq p_{\mathrm{o}}$,

i.e., the outage probability constraint (2) is satisfied.

Proof: We first present some properties of stochastic ordering that will be used later in the proof. Based on [22, Chapter 1], a real random variable $A$ is less than a random variable $B$ in the "usual stochastic order" if $\operatorname{Pr}\{A>x\} \leq$ $\operatorname{Pr}\{B>x\}$ for all $x \in(-\infty, \infty)$, which is denoted by $A \preceq B$. Two properties of stochastic ordering are

$1)$ if $u(\cdot)$ is a non-decreasing function and $A \preceq B$, then $u(A) \preceq u(B)$;

2) if $A_{i} \preceq B_{i}$ for each $i \in\{1,2, \ldots, n\}$, then $\sum_{i=1}^{n} A_{i} \preceq$ $\sum_{i=1}^{n} B_{i}$.

Now we will give the proof. By [15, Eq.(11)],

$$
\operatorname{Pr}\left\{\frac{\bar{P}_{i}^{\mathrm{r}}\left|H_{i}\right|^{2}}{\sigma^{2}+\sum_{j \neq i} \bar{S}_{j, i}^{\mathrm{r}}\left|G_{j, i}\right|^{2}}<x\right\} \leq \operatorname{Pr}\left\{\left|H_{i}\right|^{2}<\frac{x}{\bar{\gamma}_{i}}\right\}
$$

for any $x>0$. Then, for each $i=1, \ldots, E_{k^{\prime}}^{\text {all }}$ and $N_{k^{\prime}} \in \mathcal{R}_{+}$,

$$
\begin{aligned}
& \operatorname{Pr}\left\{\rho \log _{2}\left(1+\frac{\bar{P}_{i}^{\mathrm{r}}\left|H_{i}\right|^{2}}{\sigma^{2}+\sum_{j \neq i} \bar{S}_{j, i}^{\mathrm{r}}\left|G_{j, i}\right|^{2}}\right)<N_{k^{\prime}}\right\} \\
& \leq \operatorname{Pr}\left\{\rho \log _{2}\left(1+\bar{\gamma}_{i}\left|H_{i}\right|^{2}\right)<N_{k^{\prime}}\right\}
\end{aligned}
$$

due to the first property of stochastic ordering.

Finally, consider the following chain of inequalities:

$$
\begin{aligned}
& \operatorname{Pr}\left\{\sum_{i=1}^{E_{k^{\prime}}^{\text {all }}} \rho \log _{2}\left(1+\frac{\bar{P}_{i}^{\mathrm{r}}\left|H_{i}\right|^{2}}{\sigma^{2}+\sum_{j \neq i} \bar{S}_{j, i}^{\mathrm{r}}\left|G_{j, i}\right|^{2}}\right)<N_{k^{\prime}}\right\} \\
& \leq \operatorname{Pr}\left\{\sum_{i=1}^{E_{k^{\prime}}^{\mathrm{all}}} \rho \log _{2}\left(1+\bar{\gamma}_{i}\left|H_{i}\right|^{2}\right)<N_{k^{\prime}}\right\} \\
& \leq \operatorname{Pr}\left\{\sum_{i=1}^{E_{k^{\prime}}^{\mathrm{all}}} \rho \log _{2}\left(1+\bar{\gamma}_{k^{\prime}}^{\mathrm{T}}\left|H_{i}\right|^{2}\right)<N_{k^{\prime}}\right\} \\
& \leq p_{\mathrm{o}} .
\end{aligned}
$$

Here, (9) follows from the second property of stochastic ordering; (10) follows because $\bar{\gamma}_{i} \geq \bar{\gamma}_{k^{\prime}}^{\mathrm{T}}$ for all $i=1,2, \ldots, E_{k^{\prime}}^{\text {all }}$; and (11) follows from (3). This concludes the proof.

Lemma 1 allows us to map the original outage constraint (2) to the constraints on $\bar{\gamma}_{i}$, which are much easier to deal with since $\bar{\gamma}_{i}$ does not depend on fast varying CSI. Compared to the $\bar{\gamma}_{k^{\prime}}^{\mathrm{T}}$ derived in [17, Eq.(4)], the $\bar{\gamma}_{k^{\prime}}^{\mathrm{T}}$ defined in (3) is a smaller value, which implies Lemma 1 yields a better requirement 
transformation than that in [17]. By better transformation, we mean that the identical outage constraint in (2) can be guaranteed by a smaller $\bar{\gamma}_{k^{\prime}}^{\mathrm{T}}$, i.e., a less strict constraint on $\bar{\gamma}_{i}$. Note that $\bar{\gamma}_{i}$ depends on the optimization variables that will be formally defined later on. From now on, with a slight abuse of terminology, we refer to (5) as the SINR constraint.

For a given $\rho, N_{k^{\prime}}, p_{\mathrm{o}}$, and the probability density functions (pdfs) of $H_{i}$, we can treat $\bar{\gamma}_{k^{\prime}}^{\mathrm{T}}$ and $E_{k^{\prime}}^{\text {all }}$ as functions of each other. In this paper, we assume a fixed $E_{k^{\prime}}^{\text {all }}$ and then derive $\bar{\gamma}_{k^{\prime}}^{\mathrm{T}}$ from $E_{k^{\prime}}^{\text {all }}$ by, e.g., Monte Carlo (MC) simulations. It is worth mentioning that, this derivation can be conducted offline and the resulting values can be saved in a table for later use. Besides, for a network without mobility, Lemma 1 still holds, which can be shown by substituting $\left|H_{i}\right|$ and $\left|G_{j, i}\right|$ with 1 in the proof.

Similar to [4], [17], we reduce the two-dimensional RB allocation problem over both frequency and time into a sequence of one-dimensional problems in the frequency domain. Toward this end, the requirements on latency and reliability become

$$
E_{k^{\prime}}=\left\lceil E_{k^{\prime}}^{\mathrm{all}} / L_{\mathrm{tol}}\right\rceil, \quad \bar{\gamma}_{i} \geq \bar{\gamma}_{k^{\prime}}^{\mathrm{T}}, \quad \forall i=1,2, \ldots, E_{k^{\prime}},
$$

where $L_{\mathrm{tol}}$ is the latency constraint in terms of the number of scheduling time units and $E_{k^{\prime}}$ is the number of RBs allocated to V-UE $k^{\prime}$ during each scheduling time unit.

By taking the steps outlined thus far, we have transformed the V2X requirement in (2) into the constraints on $E_{k^{\prime}}$ and $\bar{\gamma}_{k^{\prime}}^{\mathrm{T}}$ with some margin, and the latter requires slowly varying CSI only. In the following, we will use $E_{k^{\prime}}$ and $\bar{\gamma}_{k^{\prime}}^{\mathrm{T}}$ in the problem formulation. Recall that these are the results from transforming the original V2X service requirement (2). In the simulations, we will explicitly evaluate the performance with respect to (2), to show the effectiveness of this transformation.

\section{B. Requirements on $C$-UEs}

In contrast to $\mathrm{V} 2 \mathrm{X}$ communications, the system usually strives to provide C-UEs with high data rates subject to some level of fairness. Therefore, the maximization of C-UEs' sum rate will be used as the optimization objective in our problem. Moreover, we employ proportional bandwidth fairness for $\mathrm{C}$ UEs [23]. As a result, the number of RBs $E_{m^{\prime}}^{\prime}$ allocated to $\mathrm{C}$-UE $m^{\prime}$ during one scheduling time unit is predetermined for all $m^{\prime} \in \mathcal{M}^{\prime}$, and $\sum_{m^{\prime}=1}^{M^{\prime}} E_{m^{\prime}}^{\prime}=F$. Since the C-UE RB allocation is orthogonal, this implies that all of the $F$ available $\mathrm{RBs}$ are allocated to the C-UEs.

\section{PRoblem Formulation}

Our problem formulation jointly deals with RRM for V-UEs and C-UEs. The performance objective is to maximize the $\mathrm{C}$ UEs' sum rate with the aforementioned fairness, subject to the V-UEs' requirements on latency and reliability, i.e., the conditions defined by (12).

For notational convenience, we introduce the concepts of sub-users and extended user sets. Specifically, we split VUE $k^{\prime}$ into $E_{k^{\prime}}$ sub-V-UEs for all $k^{\prime} \in \mathcal{K}^{\prime}$, and split C-UE $m^{\prime}$ into $E_{m^{\prime}}^{\prime}$ sub-C-UEs for all $m^{\prime} \in \mathcal{M}^{\prime}$, where each subuser corresponds to the allocation of one RB to the UE in question. Moreover, we define two extended user sets $\mathcal{K} \triangleq$

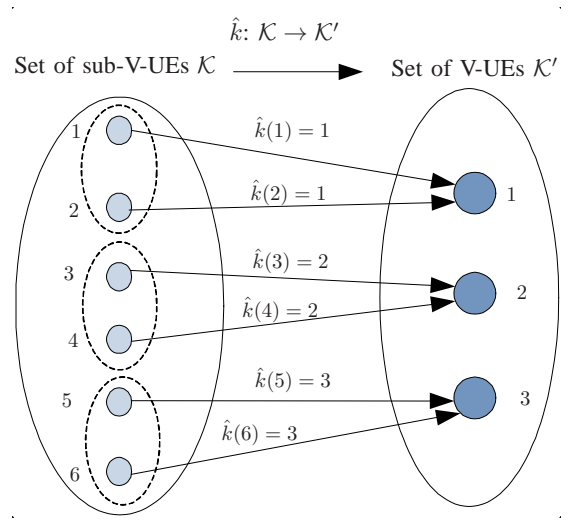

Figure 2. An example of the mapping $\hat{k}($.$) between sub-V-UEs and V-UEs,$ where $\mathcal{K}^{\prime}=\{1,2,3\}, E_{1}=E_{2}=E_{3}=2$, and $\mathcal{K}=\{1,2,3,4,5,6\}$.

$\{1,2, \ldots, K\}$ with $K=|\mathcal{K}|$ and $\mathcal{M} \triangleq\{1,2, \ldots, M\}$ with $M=|\mathcal{M}|$ for the sub-V-UEs and sub-C-UEs, respectively. To relate the original user sets and the extended user sets to each other, we define mapping $\hat{k}: \mathcal{K} \rightarrow \mathcal{K}^{\prime}$ such that $k^{\prime}=\hat{k}(k)$ is the V-UE to which sub-V-UE $k$ belongs. An example of the mapping $\hat{k}($.$) is illustrated in Fig. 2. Similarly, we define$ mapping $\hat{m}: \mathcal{M} \rightarrow \mathcal{M}^{\prime}$ such that $m^{\prime}=\hat{m}(m)$ gives the C-UE to which sub-C-UE $m$ belongs.

Based on the above definitions, the joint RRM problem is formalized below.

$$
\max _{x_{m, k}, P_{m, k}, S_{m}} \sum_{m=1}^{M} \log _{2}\left(1+\frac{S_{m} h_{\hat{m}(m)}^{\prime}}{\sigma^{2}+\sum_{k=1}^{K} P_{m, k} g_{\hat{k}(k)}}\right)
$$

subject to:

$$
\begin{aligned}
& x_{m, k} \in\{0,1\}, \quad P_{m, k} \leq P^{\max } x_{m, k} \quad \forall m, k \\
& 0 \leq P_{m, k}, \sum_{m=1}^{M} \sum_{k, \hat{k}(k)=k^{\prime}} P_{m, k} \leq P^{\mathrm{max}}, \quad \forall k^{\prime} \\
& 0 \leq S_{m}, \quad \sum_{m, \hat{m}(m)=m^{\prime}} S_{m} \leq S^{\max }, \quad \forall m^{\prime} \\
& \sum_{m=1}^{M} x_{m, k}=1, \quad \forall k \\
& \sigma^{2}+S_{m} g_{\hat{m}(m), \hat{k}(k)}^{\prime}+\sum_{l=1, l \neq k}^{K} P_{m, l} g_{\hat{k}(l), \hat{k}(k)} h_{\hat{k}(k)}
\end{aligned}
$$

$\forall m, k$

where $k \in \mathcal{K}, m \in \mathcal{M}, k^{\prime} \in \mathcal{K}^{\prime}, m^{\prime} \in \mathcal{M}^{\prime}$. In the formulation, $x_{m, k}$ is a binary variable that equals 1 if sub-V-UE $k$ is sharing the same RB with sub-C-UE $m$, and 0 otherwise, $S_{m}$ is the transmit power of sub-C-UE $m$, and $P_{m, k}$ is the transmit power of sub-V-UE $k$ on the RB shared by sub-CUE $m$. If this sharing does not take place, i.e., $x_{m, k}=0$, then $P_{m, k}$ must be 0 as well; this is implied by constraint (13b). Constraints (13c) and (13d) restrict the total transmit power for each V-UE and C-UE, respectively. Equation (13e) 
ensures that each sub-V-UE is paired with exact one sub-CUE. Finally, constraint (13f) enforces the SINR requirement for each sub-V-UE, where the left-hand side is interpreted as $\bar{\gamma}_{k}$. It should be noted that multiple sub-V-UEs are allowed to share the same RB, in contrast to the setup in [4], which restricts V-UEs to use orthogonal RBs.

The input to the problem (13) consists of the number of C-UEs $M^{\prime}$, the number of V-UEs $K^{\prime}$, the number of RBs allocated to each C-UE $E_{m^{\prime}}^{\prime}$, the number of RBs allocated to each V-UE $E_{k^{\prime}}$, the noise power $\sigma^{2}$, the max power contraint for V-UEs $P^{\max }$ and C-UEs $S^{\text {max }}$, the slow CSI $h_{m^{\prime}}^{\prime}, g_{k^{\prime}}$, $h_{k^{\prime}}, g_{m^{\prime}, k^{\prime}}^{\prime}, g_{k^{\prime}, l^{\prime}}$, and the SINR constraint $\bar{\gamma}_{k^{\prime}}^{\mathrm{T}}$. The output, represented by the optimization variables, is given by the sharing indicator $x_{m, k}$, transmit power for V-UEs $P_{m, k}$ and that for C-UEs $S_{m}$ for $k \in \mathcal{K}$ and $m \in \mathcal{M}$.

Even though (13) is an integer programming formulation, it does not prove problem complexity, since many tractable problems can be stated using integer programming models. See [24, Chapter 1] for general background on complexity theory. In the following, we formally provide and prove the main complexity result of the paper.

Theorem 1. The RB sharing and power allocation problem formalized in (13) is NP-hard.

Proof: We provide a polynomial-time reduction from the partitioning problem that is NP-complete [24, Chapter 2]. Given a set of positive integers $\left\{a_{1}, \ldots, a_{L}\right\}$, the partitioning problem is to determine if the set can be partitioned into two, such that the elements of the two subsets have equal sum. Without loss of generality, we assume $\sum_{i=1}^{L} a_{i}=2 T$ for some integer $T>0$. That is, the grand sum of all elements is even, as otherwise it is trivial to conclude infeasibility.

For any given partitioning instance, we define an RB sharing and power allocation instance, and show their polynomialtime equivalence. Let $M^{\prime}=L, E_{m^{\prime}}^{\prime}=1, \forall m^{\prime} \in \mathcal{M}^{\prime}$, and $F=L$. That is, the number of C-UEs as well as the number of available RBs both equal the number of integers in the partitioning instance, and each $\mathrm{C}$-UE has to be allocated one RB. As a result, the sets of C-UEs and sub-C-UEs coincide, i.e., $\mathcal{M}=\mathcal{M}^{\prime}$. There are two V-UEs, i.e., $K^{\prime}=2$, with $E_{1}+E_{2}=L$. This leads to $L$ sub-V-UEs. Thus $\mathcal{K}=\left\{1, \ldots, E_{1}, E_{1}+1, \ldots, L\right\}$. More specifics of $E_{1}$ (and thereby $E_{2}$ ) will be given later.

We set $S^{\max }=1$ and $P^{\max }=T$ as the max power limits of a C-UE and V-UE, respectively. The noise power $\sigma^{2}=\frac{1}{\hat{a}}$, and the SINR thresholds $\bar{\gamma}_{1}^{\mathrm{T}}=\bar{\gamma}_{2}^{\mathrm{T}}=\hat{a}$, where $\hat{a}=\max _{i=1}^{L}\left\{a_{i}\right\}$. The channel gain parameters are set as follows. For communications of own interest, $h_{m}^{\prime}=1, \forall m \in \mathcal{M}$ for the $M$ C-UEs, and $h_{1}=h_{2}=1$ for the two V-UEs. We set $g_{1}=g_{2}=0$, with the effect that the V-UEs do not cause interference to the transmission of any C-UE, Between the two V-UEs, the crosstalk links have $g_{1,2}=g_{2,1}=1$. Finally, in the direction from C-UE $m$ to the two V-UEs, $g_{m, 1}^{\prime}=g_{m, 2}^{\prime}=\frac{a_{m}-1}{\hat{a}}, \forall m \in \mathcal{M}$. By the definition of $\hat{a}$, these two values are between zero and one, and hence they qualify as channel power gains.

For the RB sharing and power allocation problem, its recognition version is to determine whether or not there exists a feasible solution such that the objective function value (13a) is no lower than some given value, which we set to $L \log _{2}(1+\hat{a})$ for the instance defined above. We derive several conclusions for feasibility and solution characterization.

First, sum rate $L \log _{2}(1+\hat{a})$ can be reached if and only if each C-UE has one RB (i.e, no C-UE will be left without being allocated any RB), and uses the maximum power $S^{\max }$ on the RB, because $M=F=L$, and the rate of a C-UE on any $\mathrm{RB}$ rate equals $\log _{2}\left(1+S_{m} /(1 / \hat{a})\right)=\log _{2}(1+\hat{a})$ if and only if $S_{m}=S^{\max }=1$. The observation is independent of resource allocation of the V-UEs because they do not generate any interference to the C-UEs. Thus, without loss of generality, we can assume C-UE $i$ is allocated $\mathrm{RB} i, i=1, \ldots, L$.

Consider the case of allowing the two V-UEs to share some RBs. Suppose two sub-V-UEs, one of each V-UE, are sharing RB $i$ with power $P^{\prime}$ and $P^{\prime \prime}$, respectively. As $g_{1,2}=g_{2,1}=1$, (13f) reads $P^{\prime} \geq a_{i}+\hat{a} P^{\prime \prime}$ and $P^{\prime \prime} \geq a_{i}+\hat{a} P^{\prime}$. As $\hat{a} \geq 1$ and $a_{i} \geq 1$, it is easy to conclude that these two inequalities cannot hold simultaneously. Therefore, RB allocation for the two VUEs is feasible only if no RB sharing occurs between them. As a result, the two V-UEs are not coupled by interference. In this case, all the $L$ RBs are used by the $L$ sub-V-UEs, with exactly one sub-V-UE on each RB. Consider any sub-V-UE, and suppose this sub-V-UE is allocated RB $i$ and hence shares the RB with C-UE $i$. Note that $S_{i}=1$ by the observation above. By (13f), in order to meet the SINR threshold, which is set to $\hat{a}=\max _{i=1}^{L}\left\{a_{i}\right\}$, the power by the sub-V-UE must be at least $\hat{a}\left(\frac{1}{\hat{a}}+\frac{a_{i}-1}{\hat{a}}\right)=a_{i}$. Recall that the $L$ sub-V-UEs originate from two V-UEs, and each of the two has power limit $T$. Thus the total power used by the $L$ sub-V-UEs together cannot be higher than $2 T$. Because $\sum_{i=1}^{L} a_{i}=2 T$, if there is a feasible solution of pairing the $L$ RBs with the $L$ sub-VUEs with one RB per sub-V-UE, the power of the sub-V-UE on RB $i$ equals $a_{i}$, and, for each V-UE, the total power of its sub-V-UEs equals the limit $T$, i.e., the solution leads to a yes-answer to the partitioning instance.

To conclude, if there is a feasible solution of the RB sharing and power allocation instance achieving total value $L \log _{2}(1+$ $\hat{a}$ ), then the power allocation of the two V-UEs' respective sub-V-UEs is a solution to the partitioning instance, such that the two subsets have cardinalities $E_{1}$ and $L-E_{1}$, and vice versa. Hence, letting parameter $E_{1}$ take values $1, \ldots, L-1$, solving the recognition version of the resulting $\mathrm{RB}$ sharing and power allocation instance is equivalent to providing the answer to the partitioning instance. As the transformation is clearly polynomial, it follows that the recognition version of the RB sharing and power allocation problem is NP-complete, and consequently its optimization version is NP-hard.

A rather common approach for RRM has been problem decomposition, in which power and RB allocation are considered separately. Indeed, if the power values are given, RB assignment becomes tractable [4] if orthogonality among VUEs is assumed. For our problem with possible RB sharing among the V-UEs, however, NP-hardness remains, even if the power levels are given a priori. This is formalized and proven in the following theorem.

Theorem 2. The RB sharing problem in (13) remains NP-hard even if the power levels of all UEs are given. 
Proof: As in the previous proof, we use the partitioning problem for polynomial-time reduction. The partitioning instance is given by a set of positive integers $\left\{a_{1}, \ldots, a_{L}\right\}$ with $\sum_{i=1}^{L} a_{i}=2 T$ for some integer $T$. Also, without loss of generality, it is assumed that $a_{i} \leq T, i=1, \ldots, L$, because otherwise the partitioning instance is trivially infeasible.

We construct an instance of the RB sharing problem with given power levels as follows. There are $K=L$ V-UEs, with $E_{k^{\prime}}=1, k^{\prime}=1, \ldots, L$, and $F=2$. That is, two RBs are to be allocated to $L$ V-UEs, each requiring one RB and thus the sets of V-UEs and sub-V-UEs coincide. For convenience, in the sequel we will stick to the sub-V-UE set $\mathcal{K}=\{1, \ldots, L\}$. There is no interference between C-UEs and V-UEs in either direction, and therefore we do not define C-UEs explicitly as they have no significance to the proof. The power of sub$\mathrm{V}$-UE $k$ is $P_{k}=a_{k}, k \in \mathcal{K}$. (Since there is no C-UE for the proof, one subscript is sufficient for the power values.) Moreover, $g_{k, l}=\frac{1}{T}, \forall k \in \mathcal{K}, l \in \mathcal{K}, k \neq l, \sigma^{2}=\frac{1}{T}$, and $h_{k}=$ $\frac{T-a_{k}+1}{a_{k} T}, \forall k \in \mathcal{K}$. As $a_{k} \leq T, 0<h_{k} \leq 1$ by construction, $\forall k \in \mathcal{K}$. The SINR threshold $\bar{\gamma}_{k^{\prime}}^{\mathrm{T}}=1, \forall k^{\prime} \in \mathcal{K}^{\prime}$.

For the defined RB sharing instance, a solution is clearly a partition of the $L$ sub-V-UEs into two subsets. The sub-V-UEs of each subset share a common RB. Denote by $\mathcal{K}_{1}$ one of the subsets, and consider an arbitrary sub-V-UE $k \in \mathcal{K}_{1}$. Using the power and channel gain values, it is easy to conclude that the SINR of $k$ reads $\frac{T-a_{k}+1}{1+\sum_{l \in \mathcal{K}_{1} \backslash\{k\}} a_{l}}$. Hence, the SINR threshold is met if and only if $\sum_{l \in \mathcal{K}_{1} \backslash\{k\}} a_{l} \leq T-a_{k}$, i.e., $\sum_{i \in \mathcal{K}_{1}} a_{i} \leq T$. Note that the condition applies to all sub-V-UEs in $\mathcal{K}_{1}$. That is, the sub-V-UEs in subset $\mathcal{K}_{1}$ can share a common RB if and only if the sum of their power values does not exceed $T$. Because the sum of all $L$ power values equals $2 T$, feasibility holds for the RB instance if and only if the power sum of the sub-V-UEs on each RB equals exactly $T$. Hence the RB sharing instance is equivalent to the partitioning instance, and the theorem follows.

Due to the NP-hardness of problem (13), we cannot expect the existence of an algorithm that is both time-efficient and guarantees global optimality, unless $\mathrm{P}=\mathrm{NP}$. Therefore, we resort to heuristic algorithms for solving the RB sharing and power allocation problem in (13).

\section{The Proposed CROWN Algorithm}

In this section, we propose the three-stage CROWN scheme to solve problem (13). To this end, we will first in Section V-A present the V-UE clustering method, where V-UEs in the same cluster do not share a common RB. Secondly, in Section V-B, we will derive the $\mathrm{RB}$ sharing algorithm which treats the clusters sequentially. Thirdly, in Section V-C, based on the RB sharing results, the transmit power for each V-UE and C-UE is further optimally adjusted taking the sum power constraints into account. Finally, how to handle the situation when the resource allocation problem is infeasible is discussed in Section V-D.

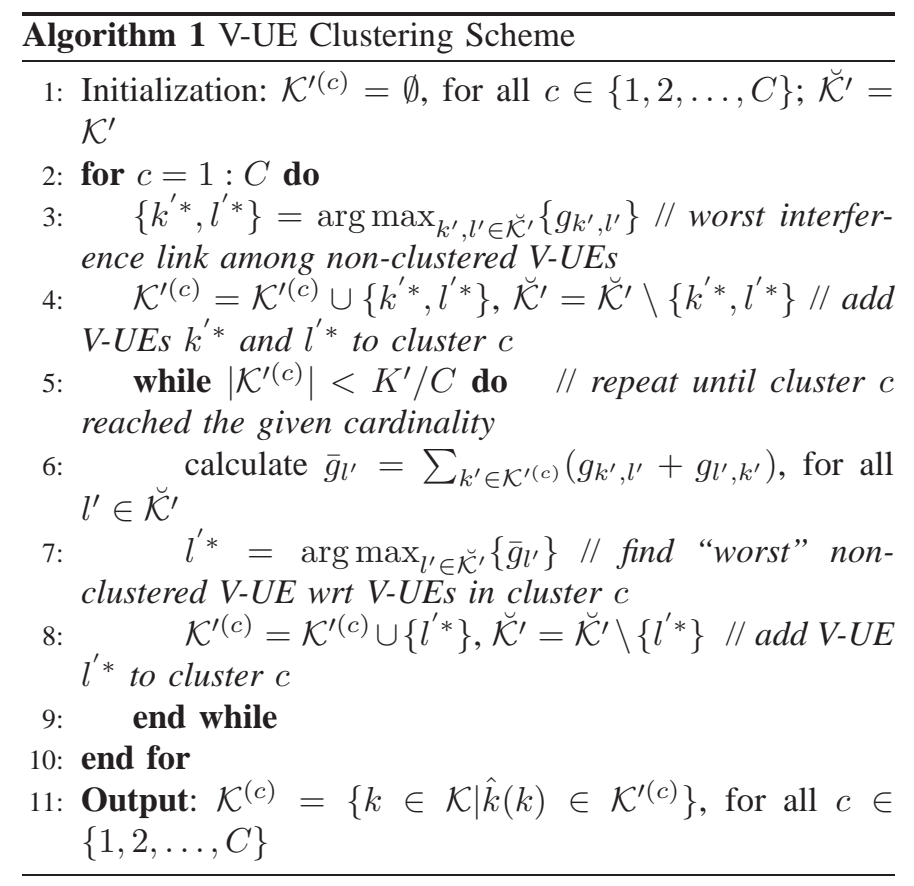

\section{A. V-UE Clustering}

In constrast to [4], Theorem 2 precludes us from using maximum weight matching (MWM) for bipartite graphs ${ }^{1}$ to achieve optimal RB sharing for problem (13), even if we separate RB and power allocation. The difficulty is caused by the potential non-orthogonality among V-UEs, which violates a prerequisite of MWM. To tackle this challenge, we could consider the V-UEs sequentially (i.e., conduct RB sharing for each V-UE one by one), since the sub-V-UEs belonging to the same V-UE cannot use the same RB. However, to utilize the optimality of MWM for bipartite graphs, we first group the $K^{\prime}$ V-UEs into $C$ clusters, where each cluster contains $K^{\prime} / C$ V-UEs ${ }^{2}$. The V-UEs in the same cluster are restricted to use orthogonal RBs, whereas the V-UEs of different clusters are allowed to share RBs.

From a performance standpoint, the V-UEs that do not share RBs in an optimum RB allocation should be grouped into one cluster. Intuitively, the more interference the V-UEs may cause to each other, the less likely it is that the V-UEs will share the same RB in an optimum allocation. By this principle, the clustering can be transformed into graph partitioning, where the V-UEs and the interference channels are interpreted as the vertices and the weighted edges, respectively. Since the graph partitioning problem is NP-hard [26], we propose a heuristic clustering method in Algorithm $1^{3}$, where the

\footnotetext{
${ }^{1}$ In graph theory, 1) bipartite graphs are used to model the scenarios in which the vertices are divided into two groups, where edges are only possible between vertices of different groups; 2) a matching is a set of edges without common vertices. Moreover, an MWM is defined as a matching where the sum of the values of the edges in the matching has a maximal value, see [25] for more details.

${ }^{2}$ For compact presentation, we here assume the number of V-UEs $K^{\prime}$ is divisible by the number of clusters $C$ and $K^{\prime} / C \geq 2$. The clustering method can easily be extended to handle the case when this assumption is not valid.

${ }^{3}$ More advanced graph partitioning algorithms can also be utilized at the price of increased complexity. However, since clustering is not the main focus of this work, detailed discussions are not presented here.
} 
notation is explained in Table I. The essential idea is, in each step, to include the V-UE that incurs the strongest sum mutual interference to all the existing V-UEs in the cluster under consideration. Upon termination, the algorithm outputs $C$ clusters of sub-V-UEs, where each cluster corresponds to $K^{\prime} / C$ V-UEs.

\section{B. RB Sharing}

Based on the clustering solution from Section V-A, we consider RB sharing for clusters $1, \ldots, C$ sequentially. Within each cluster, the RB sharing strategy will take into account the C-UEs' sum rate and the SINR constraints on the sub-VUEs belonging to both the current and previous clusters in the sequence.

Assume clusters $1, \ldots, c-1(c>1)$ have been allocated RBs, i.e., $x_{m, k}$ is known for $m \in \mathcal{M}$ and $k \in \mathcal{K}^{(1: c-1)} \triangleq$ $\mathcal{K}^{(1)} \cup \cdots \cup \mathcal{K}^{(c-1)}$, then the task is to perform optimal RB sharing for cluster $c$, i.e., decide $x_{m, k}$ for all $m \in \mathcal{M}, k \in$ $\mathcal{K}^{(c)}$. We denote by $\mathcal{K}_{m}^{(1: c-1)}$ the indices of the sub-V-UEs in $\mathcal{K}^{(1: c-1)}$ such that the RB sharing takes places with sub-C-UE $m$, i.e., $\mathcal{K}_{m}^{(1: c-1)} \triangleq\left\{k \mid k \in \mathcal{K}^{(1: c-1)}, x_{m, k}=1\right\}$.

Due to the sum power constraints (13c) and (13d), the RB sharing and power allocation problem for the sub-V-UEs in one cluster is still NP-hard [4]. For this reason, we will in this stage temporarily replace the sum power constraint on each UE with the max power constraint on each sub-UE. Specifically, for V-UE $k^{\prime}$, the max power on each of its used RBs is set to $\check{P}_{k^{\prime}}^{\max } \triangleq P^{\max } / E_{k^{\prime}}$. Likewise, for C-UE $m^{\prime}$, the max power on each of its used RBs is set to $\check{S}_{m^{\prime}}^{\max } \triangleq S^{\max } / E_{m^{\prime}}^{\prime}$. The resulting problem, which is referred to as the RB sharing problem for the sub-V-UEs in cluster $c$, is formulated as

$$
\max \sum_{m=1}^{M} \log _{2}\left(1+\frac{S_{m} h_{\hat{m}(m)}^{\prime}}{\sigma^{2}+\sum_{k, k \in \mathcal{K}^{(1: c)}} P_{m, k} g_{\hat{k}(k)}}\right)
$$

subject to:

$$
\begin{aligned}
& x_{m, k} \in\{0,1\} \quad \forall m \in \mathcal{M}, k \in \mathcal{K}^{(c)} \\
& 0 \leq P_{m, k} \leq \check{P}_{\hat{k}(k)}^{\max } x_{m, k} \quad \forall m \in \mathcal{M}, k \in \mathcal{K}^{(1: c)} \\
& 0 \leq S_{m} \leq \check{S}_{\hat{m}(m)}^{\max } \quad \forall m \in \mathcal{M} \\
& \sum_{m=1}^{M} x_{m, k}=1, \quad \forall k \in \mathcal{K}^{(c)} \\
& \sum_{k, k \in \mathcal{K}^{(c)}} x_{m, k} \in\{0,1\}, \quad \forall m \in \mathcal{M} \\
& \frac{P_{m, k} h_{\hat{k}(k)}}{\sigma^{2}+S_{m} g_{\hat{m}(m), \hat{k}(k)}^{\prime}+\sum_{l, l \in \mathcal{K}^{(1: c)}, l \neq k} P_{m, l} g_{\hat{k}(l), \hat{k}(k)}} \geq x_{m, k} \bar{\gamma}_{\hat{k}(k)}^{\mathrm{T}},
\end{aligned}
$$

where constraints (14b)-(14g) have similar interpretations as for constraints (13b)-(13f). Here the optimization variables are $S_{m}, P_{m, k}$ for all $m \in \mathcal{M}, k \in \mathcal{K}^{(1: c)}$, and $x_{m, k}$ for all $m \in \mathcal{M}, k \in \mathcal{K}^{(c)}$. However, the key output consists of $x_{m, k}$ for all $m \in \mathcal{M}, k \in \mathcal{K}^{(c)}$, whereas the power variables are subject to further adjustment in the next stage. Recall that $x_{m, k}$ for all $m \in \mathcal{M}, k \in \mathcal{K}^{(1: c-1)}$ are given. Moreover, note that the max power constraint (14c) and the SINR constraint (14g) are imposed on all the sub-V-UEs belonging to cluster $c$ and any cluster $c^{\prime}<c$.

To apply MWM for bipartite graphs to problem (14), we introduce $M-\left|\mathcal{K}^{(c)}\right|$ dummy sub-V-UEs with the associated set $\mathcal{K}_{\mathrm{D}}^{(c)}$. As a result, the model in problem (14) can be considered as a balanced bipartite graph, which means the set of sub-C-UEs $\mathcal{M}$ and the set of sub-V-UEs $\mathcal{K}_{\mathrm{A}}^{(c)} \triangleq \mathcal{K}^{(c)} \cup \mathcal{K}_{\mathrm{D}}^{(c)}$ represent the two disjoint vertex sets of equal cardinality, and $\mathcal{E}_{c} \triangleq \mathcal{M} \times \mathcal{K}_{\mathrm{A}}^{(c)}$ denotes the set of edges in the graph. Naturally, for all the dummy sub-V-UEs, we set the desired channel power gains as 0 , the interference channel power gains from and to other UEs as 0 , and the SINR constraint value as 0 . Besides, an auxiliary set is defined as $\mathcal{K}_{m, k}^{(1: c)} \triangleq \mathcal{K}_{m}^{(1: c-1)} \cup\{k\}$ for all $m \in \mathcal{M}$ and $k \in \mathcal{K}_{\mathrm{A}}^{(c)}$, which contains the sub-V-UEs that share RB with sub-C-UE $m$ if the vertexes $m$ and $k$ are connected. Furthermore, to maximize the C-UEs' sum rate, we define the edge weight $\Psi_{m, k}^{(c)}$ as the maximal achievable rate of sub-C-UE $m$ when it is sharing its RB with the sub-V-UE $k$.

For deriving the edge weight $\Psi_{m, k}^{(c)}$, we define some relevant quantities for a set of sub-V-UEs being assumed to share the same RB with sub-C-UE $m$. Take the set $\mathcal{K}_{m, k}^{(1: c)}$ as an example, i.e., assume the sub-V-UEs in $\mathcal{K}_{m, k}^{(1: c)}$ are sharing the same RB with sub-C-UE $m$. We use $k_{i}$ to denote the sub-V-UE index that corresponds to the $i$ th element in the set $\mathcal{K}_{m, k}^{(1: c)}$. Moreover, we define matrix $\boldsymbol{\Omega}^{(m)} \in \mathbb{R}^{\left|\mathcal{K}_{m, k}^{(1: c)}\right| \times\left|\mathcal{K}_{m, k}^{(1: c)}\right|}$, and vectors $\boldsymbol{g}^{(m)}$, $\boldsymbol{\mu}^{(m)}, \boldsymbol{\theta}^{(m)}$, and $\boldsymbol{p}^{(m), \max }$ with dimension $\left|\mathcal{K}_{m, k}^{(1: c)}\right|$, where

$$
\begin{aligned}
& \Omega_{i, j}^{(m)} \triangleq \begin{cases}\bar{\gamma}_{\hat{k}\left(k_{i}\right)}^{\mathrm{T}} g_{\hat{k}\left(k_{j}\right), \hat{k}\left(k_{i}\right)} / g_{\hat{k}\left(k_{i}\right)} & \text { if } i \neq j \\
0 & \text { otherwise, }\end{cases} \\
& g_{i}^{(m)} \triangleq g_{\hat{k}\left(k_{i}\right)} \\
& \mu_{i}^{(m)} \triangleq g_{\hat{m}(m), \hat{k}\left(k_{i}\right)}^{\prime} \bar{\gamma}_{\hat{k}\left(k_{i}\right)}^{\mathrm{T}} \\
& \theta_{i}^{(m)} \triangleq \sigma^{2} \bar{\gamma}_{\hat{k}\left(k_{i}\right)}^{\mathrm{T}} \\
& p_{i}^{(m), \max } \triangleq \check{P}_{\hat{k}\left(k_{i}\right)}^{\max } .
\end{aligned}
$$

Note that for different sets of sub-V-UEs, the definitions of $k_{i}, \boldsymbol{\Omega}^{(m)}, \boldsymbol{g}^{(m)}, \boldsymbol{\mu}^{(m)}, \boldsymbol{\theta}^{(m)}$, and $\boldsymbol{p}^{(m), \max }$ have the same interpretations, but vary in dimensions and values.

Lemma 2. For all sub-C-UE $m \in \mathcal{M}$ and sub-V-UE $k \in$ $\mathcal{K}_{\mathrm{A}}^{(c)}$, the edge weight $\Psi_{m, k}^{(c)}$, which is defined as the maximal achievable rate of sub-C-UE $m$ when sharing its RB with sub$\mathrm{V}$-UE $k$, is given as

$$
\Psi_{m, k}^{(c)}=\left\{\begin{array}{c}
\log _{2}\left(1+\frac{\check{S}_{\hat{m}(m)}^{\max } h_{\hat{m}(m)}^{\prime}}{\sigma^{2}}\right) \quad \text { if } k \in \mathcal{K}_{\mathrm{D}}^{(c)}(20 \mathrm{a}) \\
\log _{2}\left(1+\frac{\check{S}_{m}^{*} h_{\hat{m}(m)}^{\prime}}{\sigma^{2}+\boldsymbol{\alpha}^{\top} \boldsymbol{g}^{(m)} \check{S}_{m}^{*}+\boldsymbol{\beta}^{\top} \boldsymbol{g}^{(m)}}\right) \text { if } k \in \mathcal{K}^{(c)}, \\
\left(\boldsymbol{I}-\boldsymbol{\Omega}^{(m)}\right)^{-1} \geq \mathbf{0}, \text { and } \boldsymbol{\beta} \leq \boldsymbol{p}^{(m), \max } \\
-\infty
\end{array}\right.
$$


where $\boldsymbol{\alpha} \triangleq\left(\boldsymbol{I}-\boldsymbol{\Omega}^{(m)}\right)^{-1} \boldsymbol{\mu}^{(m)}, \boldsymbol{\beta} \triangleq\left(\boldsymbol{I}-\boldsymbol{\Omega}^{(m)}\right)^{-1} \boldsymbol{\theta}^{(m)}$,

$$
\check{S}_{m}^{*}=\min \left\{\check{S}_{\hat{m}(m)}^{\max },\left\{\left(p_{i}^{(m), \max }-\beta_{i}\right) / \alpha_{i}\right\}_{i=1}^{\left|\mathcal{K}_{m, k}^{(1: c)}\right|}\right\},
$$

and $\boldsymbol{\Omega}^{(m)}, \boldsymbol{g}^{(m)}, \boldsymbol{\mu}^{(m)}, \boldsymbol{\theta}^{(m)}$, and $\boldsymbol{p}^{(m), \max }$ are given by (15)(19), respectively.

Proof: For each dummy sub-V-UE $k \in \mathcal{K}_{\mathrm{D}}^{(c)}$, the edge weight $\Psi_{m, k}^{(c)}$, i.e., the maximal achievable rate of sub-C-UE $m$, is equal to (20a), since there is no SINR constraint on sub-V-UE $k$ and there is no interference from sub-V-UE $k$ to sub-C-UE $m$.

On the other hand, for each non-dummy sub-V-UE $k \in$ $\mathcal{K}^{(c)}, \Psi_{m, k}^{(c)}$ is the optimum of the maximization problem (22).

$$
\max _{S_{m}, \boldsymbol{p}^{(m)}} \log _{2}\left(1+\frac{S_{m} h_{\hat{m}(m)}^{\prime}}{\sigma^{2}+\boldsymbol{p}^{(m) \top} \boldsymbol{g}^{(m)}}\right)
$$

subject to:

$$
\begin{aligned}
& \mathbf{0} \leq \boldsymbol{p}^{(m)} \leq \check{\boldsymbol{p}}^{(m), \max } \\
& 0 \leq S_{m} \leq \check{S}_{\hat{m}(m)}^{\max }, \\
& \left(\boldsymbol{I}-\boldsymbol{\Omega}^{(m)}\right) \boldsymbol{p}^{(m)} \geq \boldsymbol{\mu}^{(m)} S_{m}+\boldsymbol{\theta}^{(m)}
\end{aligned}
$$

where $\boldsymbol{p}^{(m)}$ stacks the power values $P_{m, k}$ with $p_{i}^{(m)} \triangleq P_{m, k_{i}}$ for all $i \in \mathcal{K}_{m, k}^{(1: c)}$, and (22d) is a matrix form of the SINR constraints on sub-V-UEs.

Consider the constraint in (22d). As shown by [27], a necessary and sufficient condition for (22d) to have a positive solution $\boldsymbol{p}^{(m)}$ for every positive vector $\boldsymbol{\mu}^{(m)} S_{m}+\boldsymbol{\theta}^{(m)}$ is that $\left(\boldsymbol{I}-\boldsymbol{\Omega}^{(m)}\right)^{-1}$ is nonnegative for $\boldsymbol{\Omega}^{(m)} \geq \mathbf{0}$. In addition, due to the max power constraint (22b), problem (22) is feasible if and only if $\left(\boldsymbol{I}-\boldsymbol{\Omega}^{(m)}\right)^{-1} \geq \mathbf{0}$ and $\boldsymbol{\beta} \leq \boldsymbol{p}^{(m), \max }$ [28]. Therefore, $\left(\boldsymbol{I}-\boldsymbol{\Omega}^{(m)}\right)^{-1}$ combined with $\boldsymbol{\beta}$ can be used as a metric to determine the feasibility of RB sharing between sub-C-UE $m$ and sub-V-UE $k$. More specifically, if $\left(\boldsymbol{I}-\boldsymbol{\Omega}^{(m)}\right)^{-1} \geq \mathbf{0}$ and $\boldsymbol{\beta} \leq \boldsymbol{p}^{(m), \max }$, the edge between $m$ and $k$ represents feasible RB sharing of the two sub-UEs and its weight $\Psi_{m, k}^{(c)}$ equals the maximum of problem (22); otherwise, RB sharing of $m$ and $k$ is infeasible and we disable this edge by setting $\Psi_{m, k}^{(c)}=-\infty$ so that the edge between $m$ and $k$ will not be selected.

Now the task is to solve problem (22) for the case (I$\left.\boldsymbol{\Omega}^{(m)}\right)^{-1} \geq \mathbf{0}$ and $\boldsymbol{\beta} \leq \boldsymbol{p}^{(m), \text { max }}$. Since the objective function (22a) is nonincreasing in terms of each element of $\boldsymbol{p}^{(m)}$, the solution $\check{\boldsymbol{p}}^{(m) *}$ satisfying the equality in (14g) must be optimal to problem (22). By solving this equality, we obtain

$$
\check{\boldsymbol{p}}^{(m) *}=\left(\boldsymbol{I}-\boldsymbol{\Omega}^{(m)}\right)^{-1}\left(\boldsymbol{\mu}^{(m)} S_{m}+\boldsymbol{\theta}^{(m)}\right) \triangleq \boldsymbol{\alpha} S_{m}+\boldsymbol{\beta} .
$$

Due to the nonnegativity of $\left(\boldsymbol{I}-\boldsymbol{\Omega}^{(m)}\right)^{-1}$, we have $\boldsymbol{\alpha} \geq \mathbf{0}$ and $\boldsymbol{\beta} \geq \mathbf{0}$. Then, by substituting the expression of $\check{\boldsymbol{p}}^{(m) *}$ into (22a), we eliminate $\boldsymbol{p}^{(m)}$ and transform the objective function into

$$
(22 \mathrm{a})=\max \log _{2}\left(1+\frac{S_{m} h_{\hat{m}(m)}^{\prime}}{\sigma^{2}+\boldsymbol{\alpha}^{\top} \boldsymbol{g}^{(m)} S_{m}+\boldsymbol{\beta}^{\top} \boldsymbol{g}^{(m)}}\right) .
$$

From the first derivative of (24) with respect to $S_{m}$, it can be easily verified that (24) is nondecreasing in $S_{m} \geq 0$. Hence, by also taking into account constraints in (22b) and (22c), the maximum of problem (22) can be reached at the power value $\check{S}_{m}^{*}$ in (21). Finally, substituting $\check{S}_{m}^{*}$ into (24), we obtain the optimum of problem (22) as given by (20b). This concludes the proof.

With the vertex sets $\mathcal{M}$ and $\mathcal{K}_{\mathrm{A}}^{(c)}$, as well as the established weights $\Psi_{m, k}^{(c)}$ from Lemma 2, problem (14) is in fact a MWM problem for bipartite graphs and can be reformulated as

$$
\max _{x_{m, k}} \sum_{m \in \mathcal{M}, k \in \mathcal{K}_{\mathrm{A}}^{(c)}} x_{m, k} \Psi_{m, k}^{(c)}
$$

subject to:

$$
\begin{gathered}
x_{m, k} \in\{0,1\}, \quad \forall m \in \mathcal{M}, k \in \mathcal{K}_{\mathrm{A}}^{(c)} \\
\sum_{m, m \in \mathcal{M}} x_{m, k}=1, \quad k \in \mathcal{K}_{\mathrm{A}}^{(c)} \\
\sum_{k, k \in \mathcal{K}_{\mathrm{A}}^{(c)}} x_{m, k}=1, \quad \forall m \in \mathcal{M} .
\end{gathered}
$$

We can use the Hungarian algorithm [25] to efficiently solve the problem (25), and thereby obtain the optimal RB sharing indicator solution $x_{m, k}^{*}$. The number of operations is upperbounded by $O\left(F^{3}\right)$ [25] (recall that the total number of RBs is $F=|\mathcal{M}|$ ). Moreover, if the maximum of problem (25) is $-\infty$, we consider both problem (14) and the original problem (13) infeasible.

Until now, we have optimally solved problem (14) and obtained the RB sharing results $x_{m, k}^{*}$ for the sub-V-UEs belonging to cluster $c$. The algorithmic steps for all the $C$ clusters are presented in Algorithm 2, where lines 3-8 describe the RB assignment procedure for cluster $c$.

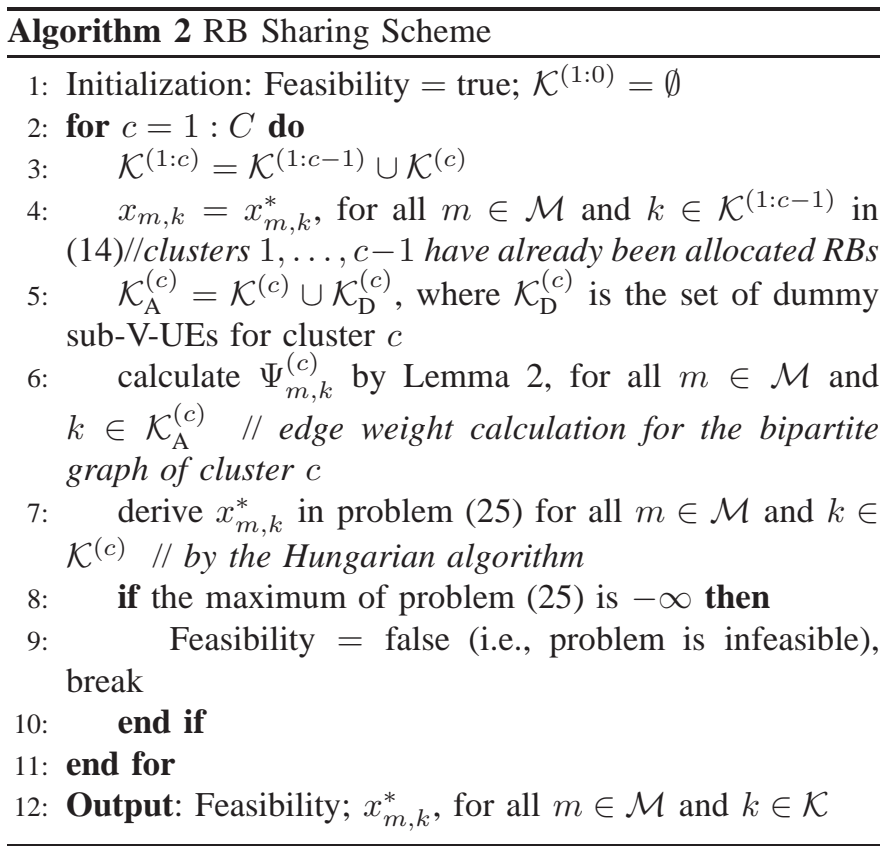




\section{Power Allocation}

If the problem is reported feasible by Algorithm 2, the third stage of the CROWN is to allocate power, which is referred to as the power allocation problem (PAP). In PAP, the sum power constraints (13c) and (13d) for each UE are considered, instead of individual power limits of the sub-UEs. Based on the $\mathrm{RB}$ sharing results from Algorithm 2, we denote by $\mathcal{K}_{m}^{*} \triangleq$ $\left\{k \mid k \in \mathcal{K}, x_{m, k}^{*}=1\right\}$, for all $m \in \mathcal{M}$, the indices of the sub-V-UEs such that RB sharing takes places with sub-C-UE $m$. Then, for $\mathcal{K}_{m}^{*}$, the quantities defined in (15)-(18) can be obtained, here denoted by $\boldsymbol{\Omega}^{(m) *}, \boldsymbol{g}^{(m) *}, \boldsymbol{\mu}^{(m) *}$, and $\boldsymbol{\theta}^{(m) *}$. Assuming feasibility in Algorithm 2, i.e., $\left(\boldsymbol{I}-\boldsymbol{\Omega}^{(m) *}\right)^{-1} \geq \mathbf{0}$ and $\left(\boldsymbol{I}-\boldsymbol{\Omega}^{(m) *}\right)^{-1} \boldsymbol{\theta}^{(m) *} \leq \boldsymbol{p}^{(m), \max }$, the PAP is formulated as follows.

$$
\max _{P_{m, k}, S_{m}} \sum_{m=1}^{M} \log _{2}\left(1+\frac{S_{m} h_{\hat{m}(m)}^{\prime}}{\sigma^{2}+\sum_{k, k \in \mathcal{K}_{m}^{*}} P_{m, k} g_{\hat{k}(k)}}\right)
$$

subject to:

(13c), (13d)

$$
\left(\boldsymbol{I}-\boldsymbol{\Omega}^{(m) *}\right) \boldsymbol{p}^{(m)} \geq \boldsymbol{\mu}^{(m) *} S_{m}+\boldsymbol{\theta}^{(m) *}, \quad \forall m \in \mathcal{M}
$$

where $p_{i}^{(m)} \triangleq P_{m, k_{i}}$ for all $i \in\left\{\mathcal{K}_{m}^{*}\right\}$.

Since the objective function (26a) is not concave with respect to $P_{m, k}$, the PAP is not a convex problem. Nevertheless, by the same argument used when deriving (23), we know that the solution in the form of

$$
\boldsymbol{p}^{(m) *}=\left(\boldsymbol{I}-\boldsymbol{\Omega}^{(m) *}\right)^{-1}\left(\boldsymbol{\mu}^{(m) *} S_{m}+\boldsymbol{\theta}^{(m) *}\right),
$$

must be an optimal solution to problem (26). In other words, for all $k \in \mathcal{K}_{m}^{*}$, the optimal power $P_{m, k}^{*}$ can be expressed as an affine function of $S_{m}$ with nonnegative slope and offset. Furthermore, by substituting $P_{m, k}^{*}$ into (26a), problem (26) is transformed into an equivalent convex problem, where the convexity can be shown through verifying the positive semidefinite property of the Hessian matrix of the objective function. The transformed problem can then be solved by an interior point method to obtain the optimal power $S_{m}^{*}$ for subC-UE $m$.

Moreover, the CROWN algorithm has much lower complexity compared to solving (13) optimally by an exhaustive search that has exponentially increasing complexity with respect to the problem size. Specifically, in the CROWN scheme, the computations of the first two steps, i.e., V-UE clustering and $\mathrm{RB}$ sharing, are upper-bounded by $O\left(K^{\prime 2}\right)$ and $O\left(C F^{3}\right)$, respectively. Moreover, the third step of power allocation, which is achieved by an interior point method, is quite efficient in practice and its complexity hardly grows with the problem size [29, p. 595]. The overall polynomial-time efficiency of the CROWN scheme promotes its practical relevance.

\section{What if the Problem Is Infeasible?}

Given the fact that wireless communication systems are not designed for full reliability at any cost, infeasibility ${ }^{4}$ is a

\footnotetext{
${ }^{4}$ This infeasibility can be caused by either the problem itself (e.g., problem (13)) or the limitations of the algorithms. Unfortunately, deciding the feasibility of problem (13) is NP-hard. Thus, we will not distinguish between the sources of infeasibility here.
}

possible outcome of the RRM context that we consider. Due to the stringent requirement of safety-critical V2X communications, it is of paramount importance to notify the application about the absence of reliability. Hence, as in [30], [31], we can use an Availability Indicator to include the feasibility information. More specifically, the required reliability of V2X communication (in terms of (13f)) is declared to be available if a RRM scheme leads to feasible solution and to be unavailable otherwise.

\section{Vi. PERformance Evaluation}

\section{A. Scenarios and Parameters}

We assume a single cell outdoor system with a carrier frequency of $800 \mathrm{MHz}$ and that each RB has a bandwidth of $180 \mathrm{kHz}$. In particular, we consider test case test case 2 defined by METIS $^{5}$ [32]. The case uses an urban environmental model similar to the Manhattan grid layout. The channel models are specified in [32], which describes large scale modeling for various propagations scenarios (PSs). We will use PS\#3 in [32] for the links connected to the eNB (i.e., $h_{m^{\prime}}^{\prime}$ and $g_{k^{\prime}}^{\prime}$ ) and PS\#9 in [32] for the links between UEs (i.e., $h_{k^{\prime}}$ and $\left.g_{m^{\prime} k^{\prime}}\right)$.

Simulation parameters are summarized as follows: the maximum transmit powers are $P^{\max }=S^{\max }=24 \mathrm{dBm}$ and each RB carries $\rho=84$ complex symbols. The antenna height is $26 \mathrm{~m}$ at the eNB and $1.5 \mathrm{~m}$ at each UE. The intended broadcast range of each V-UE is $50 \mathrm{~m}$. Also, the noise floor is assumed to be $-117 \mathrm{dBm}$ at the eNB and each V-UE. The fast fading effects of channels are assumed to be Rayleigh distributed with unit power gain and independent over the RBs. Finally, one scheduling time unit (i.e., the time period of one RB) is $0.5 \mathrm{~ms}$ and the period of CSI reports from V-UEs is $100 \mathrm{~ms}$.

\section{B. Baseline Methods}

We consider CROWN as well as its simplified version without the last power allocation stage, which is denoted as CROWN-noPA. In the CROWN-noPA, $S_{m}^{*}$ and $P_{m, k}^{*}$, for all $m \in \mathcal{M}$ and $k \in \mathcal{K}$, are set as the corresponding values that achieve the optimum when solving problem (14) for cluster $C$. We compare the CROWN and CROWN-noPA with the following schemes.

1) [12]-Ext. In [12], a three-step scheme is derived to maximize the sum rate of C-UEs and V-UEs with SINR constraints on both C-UEs and V-UEs, where different V-UEs cannot share a common RB.To apply this scheme to the D2Dsupported V2X framework proposed here, we have made some adaptations. Firstly, we introduce sub-C-UE and sub-V-UE to model the candidate RBs for one UE. Correspondingly, the max power constraints become $\check{S}_{m^{\prime}}^{\max }$ and $\check{P}_{k^{\prime}}^{\max }$ for each sub$\mathrm{C}-\mathrm{UE}$ and each sub-V-UE, respectively. Moreover, we change the objective from maximizing the sum rate of both C-UEs and V-UEs into maximizing the sum rate of C-UEs, and drop the

\footnotetext{
${ }^{5}$ In addition to METIS, 3GPP also defines D2D proximity service environments. However, the setup given by $3 \mathrm{GPP}$ is for a generic D2D public safety scenario, but with no specifics for vehicular communications yet. Therefore, we leave the evaluation of the proposed CROWN scheme in 3GPP scenarios for future work.
} 
SINR constraints on C-UEs. Finally, we perform the scheme for each V-UE sequentially to allow for non-orthogonality among the V-UEs.

2) RBSPA in [17] which solves problem (13) in two stages. Firstly, ignoring the max power constraints for both V-UEs and $\mathrm{C}$-UEs, RBs are assigned to sub-V-UEs according to a specifically designed metric that relies on Perron-Frobenius theory. Secondly, based on the RB sharing outcome of stage one, the transmit power for each V-UE and C-UE is optimally adjusted by considering the sum power constraints.

3) The global optimal solution to problem (13), found by the exhaustive search over all the RB sharing possibilities. Due to its exponentially increased complexity, the approach is applied only for the case of $F=4$, i.e., 4 RBs in total during each scheduling time unit.

\section{Performance Metrics}

Following the similar ideas of the performance metrics proposed in [21], i.e., service reliability, service availability, and service failure, we base our evaluation on four metrics: 1) probability of availability ( $\mathrm{PoA})$, where "availability" is defined in Section V-D; 2) sum rate of C-UEs when fast fading is disregarded (i.e., value of the objective in (13)); 3) cumulative distribution function (CDF) of the C-UEs' sum rate; 4) CDF of one V-UE's transmitted bits within $5 \mathrm{~ms}$, i.e., the left-hand side of the inner inequality in (1). Note that, as explained in Section III-A, the transmitted data from VUEs is assumed to span over the entire time period, i.e., $5 \mathrm{~ms}$ in our simulations. Hence, as long as the RRM algorithms declare availability, all V-UEs achieve a delay of $5 \mathrm{~ms}$ with a probability exceeding $1-p_{o}$.

The following results are obtained from 500 random network instances. The calculations of the last three metrics are conditional on availability. The second metric is the averaged result over the 500 network instances. The last two metrics are evaluated when the randomness from both network realizations and fast fading effects is involved in simulations.

\section{Simulation Results}

From the requirements given by METIS [2], we have $N_{k^{\prime}}=12800$ bits, $p_{\mathrm{o}}=10^{-5}$ (i.e., a transmission reliability of $99.999 \%$ ), and $L_{\text {tol }}=10$ (i.e., a latency requirement of $5 \mathrm{~ms}$ ). As analyzed in Section III-A, the relationship between $E_{k^{\prime}}^{\text {all }}$ and $\bar{\gamma}_{k^{\prime}}^{\mathrm{T}}$ can be derived from (3) through a MC method. Then $E_{k^{\prime}}$ can be calculated via (12). This way, the combinations of $\left\{E_{k^{\prime}}, \bar{\gamma}_{k^{\prime}}^{\mathrm{T}}\right\}$ that have been used are $\{2,1406.6\},\{3,162.5037\},\{4,51.0853\}, \quad\{5,24.2406\}$, $\{6,14.2085\}, \quad\{7,9.4319\}, \quad\{8,6.8325\}, \quad\{9,5.2168\}$, $\{10,4.1500\}, \quad\{12,2.8682\}, \quad\{14,2.1471\}, \quad\{16,1.6891\}$, $\{18,1.3862\}, \quad\{20,1.1645\}, \quad\{22,1.0009\}, \quad\{24,0.8751\}$, $\{26,0.7751\},\{28,0.6947\}$, and $\{30,0.6289\}$, where the values of $\bar{\gamma}_{k^{\prime}}^{\mathrm{T}}$ are in linear scale. Comparing the combinations here with that in [17], improved requirement transformation proposed in Lemma 1 is demonstrated.

Fig. 3 compares the C-UEs' sum rates of the RRM schemes when $F=4$ and shows the performance gap with respect to the global optimum. Recall that the values shown in the labels

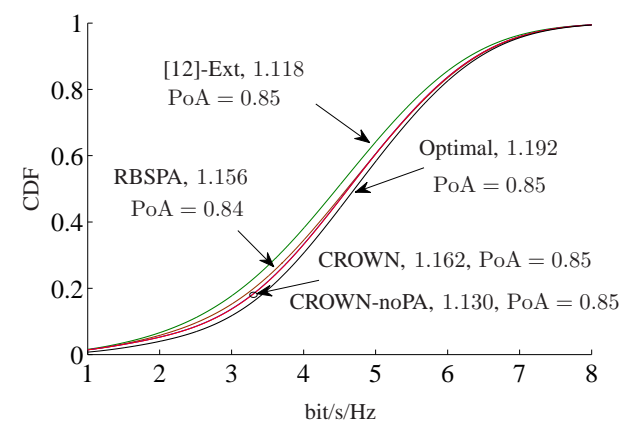

Figure 3. Sum rate of C-UEs with $F=4, M^{\prime}=4, K^{\prime}=2, E_{m^{\prime}}^{\prime}=1$, $E_{k^{\prime}}=2$, and $C=2$.

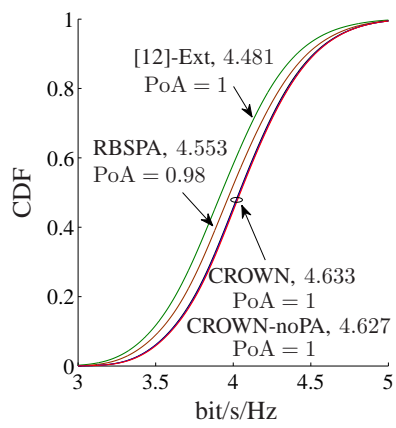

(a)

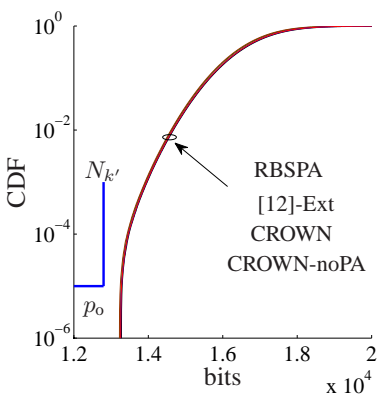

(b)
Figure 4. $F=100, M^{\prime}=25, K^{\prime}=30, E_{m^{\prime}}^{\prime}=4, E_{k^{\prime}}=6$, and $C=10$. (a) Sum rate of C-UEs. (b) Number of bits that could be transmitted within $5 \mathrm{~ms}$ for each V-UE.

are based on the long-term CSI, i.e., the performance metric 2 ), whereas the CDF curves represent results for which fast fading effects are accounted for, i.e., performance metric 3). By comparing the values to the $\mathrm{CDFs}$, it can be seen that even though the RRM decisions are based on long-term CSI, the results remain reasonably accurate for realistic channel conditions with fast fading. Moreover, we note that PoA = 0.85 even for the optimal solution, which illustrates the fact that the unavailability could be inevitable due to unfavorable scenarios. Besides, the performance difference of all the RRM schemes to optimum is small in this toy example. Especially, the deviation of CROWN and CROWN-noPA from the optimal solution is very small.

Now consider the more realistic scenario with $F=100$ RBs per scheduling time unit. We define the traffic load as the number of V-UEs. With medium load, i.e., $K^{\prime}=30$, Fig. 4(a) and Fig. 4(b) show the performance of C-UEs and V-UEs, respectively. In Fig. 4(a), the CDFs of the C-UEs sum rates are plotted. It is again observed that the long-term RRM schemes lead to valid results even when fast fading effects are present. Moreover, CROWN and CROWN-noPA perform better than [12]-Ext and RBSPA. Fig. 4(b) depicts the CDFs of the number of bits that could be transmitted within $5 \mathrm{~ms}$ for one V-UE when problem (13) is declared feasible. The four CDF curves are below the point $\left(N_{k^{\prime}}, p_{\mathrm{o}}\right)$, implying that the V-UE QoS requirement (2) is indeed fulfilled by all the four schemes. Note, however, that the V-UEs have only $N_{k^{\prime}}$ bits to transmit. Hence, the curves in Fig. 4(b) simply say that the 


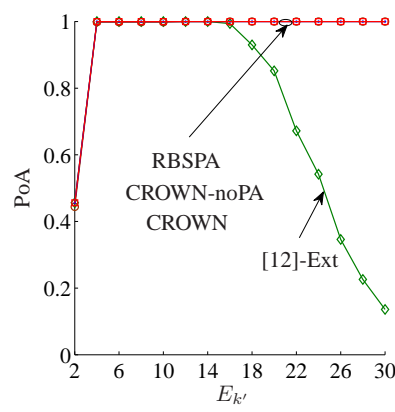

(a)

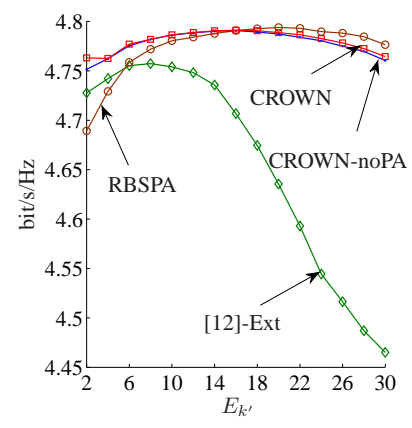

(b)
Figure 5. $F=100, K^{\prime}=10, M^{\prime}=25, E_{m^{\prime}}^{\prime}=4$, and $C=10$. (a) Availability. (b) Sum rate of C-UEs.

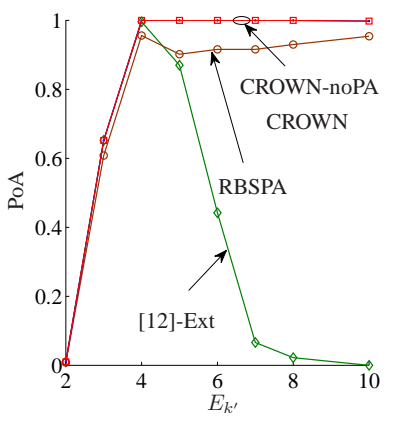

(a)

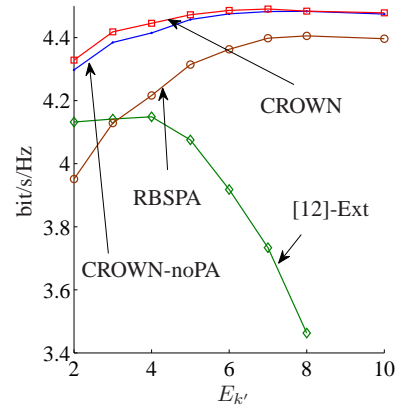

(b)
Figure 6. $F=100, K^{\prime}=50, M^{\prime}=25, E_{m^{\prime}}^{\prime}=4$, and $C=10$. (a) Availability. (b) Sum rate of C-UEs.

V-UEs succeed in transmitting $N_{k^{\prime}}$ bits with a probability that exceeds $1-p_{o}$, whenever the RRM problem is feasible.

Next, we evaluate the RRM schemes based on a palette of factors such as $E_{k^{\prime}}, K^{\prime}$, and $C$.

1) Effect of $E_{k^{\prime}}$ : Note that $E_{k^{\prime}}$ is an input to the RRM algorithms and has a direct impact on the SINR constraint $\bar{\gamma}_{k^{\prime}}^{\mathrm{T}}$, as explained above. Fig. 5 plots the PoA and C-UEs sum rate for $K^{\prime}=10$ with $0 \leq E_{k^{\prime}} \leq 30$. Concerning availability, both RBSPA and the proposed schemes are robust to various $E_{k^{\prime}}$. On the other hand, the PoA of [12]-Ext drops significantly after $E_{k^{\prime}}>16$. This dramatic degradation also appears in the rate curves for [12]-Ext, as shown in Fig. 5(b). Recall that we employ a straightforward extension to apply the scheme in [12], which is devised for orthogonal V-UE model, to the setup where non-orthogonal V-UEs are allowed. The unsatisfactory performance of [12]-Ext reveals the ineffectiveness of the simple adaptation and implies the necessity of the specific RRM design when allowing multiple V-UEs to share the same RB. Besides, for this low load scenario, i.e., $K^{\prime}=10$, the rate difference between RBSPA and CROWN (as well as CROWNnoPA) is quite small, as observed from Fig. 5(b).

Results for the case when the number of V-UEs $K^{\prime}$ is increased to 50 are depicted in Fig. 6. The poor performance of [12]-Ext is again revealed here. Moreover, in this situation, RBSPA has obviously worse performance than CROWN and CROWN-noPA. This is due to the limitations of RBSPA: 1) its $\mathrm{RB}$ sharing step ignores the max power constraint for each $\mathrm{UE}$; 2) the metric for RB allocation is based on the spectral

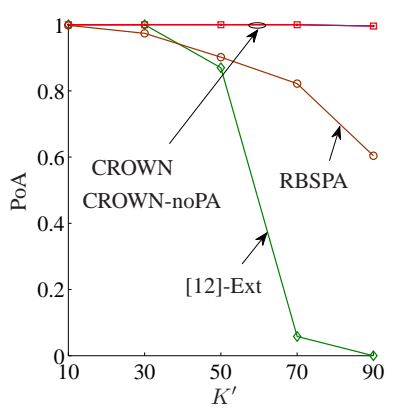

(a)

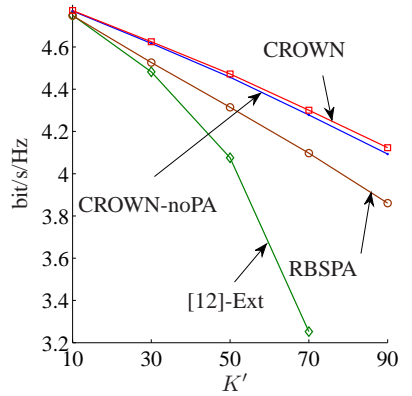

(b)
Figure 7. $F=100, M^{\prime}=25, E_{m^{\prime}}^{\prime}=4, E_{k^{\prime}}=5$, and $C=10$. (a) Availability. (b) Sum rate of C-UEs.

radius which, however, does not perfectly optimize the C-UE rates. Finally, we note from Fig. 6(b) that CROWN performs slightly better than CROWN-noPA.

To summarize, the choice of $E_{k^{\prime}}$ will indeed affect the performance of the RRM schemes. In general, increasing $E_{k^{\prime}}$ gives less required transmit power of the V-UEs on the allocated RBs due to the lowered $\bar{\gamma}_{k^{\prime}}^{\mathrm{T}}$, which then leads to increased rate of the $\mathrm{C}$-UE for which $\mathrm{RB}$ sharing takes place. On the other hand, increasing $E_{k^{\prime}}$ implies that more C-UE RBs suffer from interference caused by V-UEs. Therefore, the optimal $E_{k^{\prime}}$ is not fixed and usually depends on the considered scheme as well as the traffic load. Nevertheless, two conclusions can be drawn here. Firstly, $E_{k^{\prime}}=2$ and $E_{k^{\prime}}=3$ are often poor choices since the corresponding $\bar{\gamma}_{k^{\prime}}^{\mathrm{T}}$ may be too aggressive. Secondly, the proposed CROWN and CROWN-noPA have a relatively wide range of acceptable $E_{k^{\prime}}$. Based on our observations, setting $E_{k^{\prime}}$ in interval $[4,8]$ works well for most traffic load scenarios, e.g., $0<K \leq 90$.

2) Effect of $K^{\prime}$ : The RRM methods are evaluated in Fig. 7 with respect to $K^{\prime}$ for $E_{k^{\prime}}=5$. It can be observed that the [12]-Ext performance in both PoA and sum rate drops significantly when $K^{\prime} \geq 50$. Moreover, as seen in Fig. 7(a), the PoA of the RBSPA degrades notably with increased $K^{\prime}$, while the PoAs of the CROWN and CROWN-noPA show strong robustness. Concerning the sum rate of the C-UEs, not surprisingly, the performance of all the RRM schemes deteriorates with larger $K^{\prime}$ as shown in Fig. 7(b). Compared to other algorithms, however, the CROWN schemes exhibits superiority, especially for high load scenario.

3) Effect of $C$ : The results in Fig. 4 to Fig. 7 assume that $C=10$ clusters are used. Now we assess the influence of $C$ on CROWN and CROWN-noPA. To simplify the presentation, we define the number of V-UEs per cluster as $\Phi \triangleq K^{\prime} / C$. For $K^{\prime}=60$, Fig. 8 plots the C-UEs sum rate versus $\Phi$. For these settings, both algorithms have a PoA that is equal to 1.0 for the considered range of $\Phi$. Hence, the PoA is not shown in the figure. As seen, the sum rate is first improved and then degraded with increased $\Phi$. In fact, the smaller the $\Phi$ is, the more the spatial reuse gain can be obtained, but at the same time, a smaller $\Phi$ results in larger loss of the optimality of MWM. Hence, the optimum of $\Phi$ is a tradeoff between the two factors and obviously depends on $K^{\prime}$. By the scale of the y-axis in Fig. 8, we observe that the difference in sum rate is 


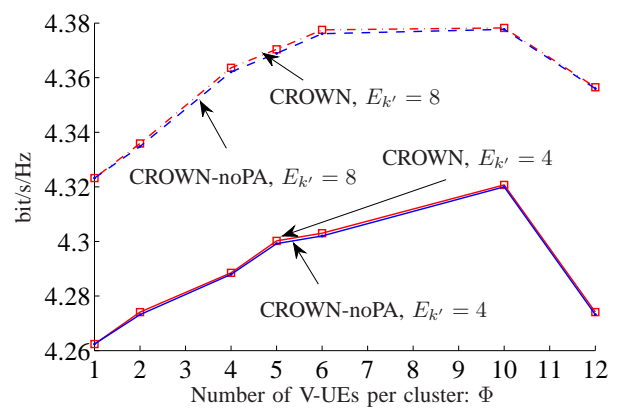

Figure 8. Sum rate of C-UEs. $F=100, K^{\prime}=60, M^{\prime}=25$, and $E_{m^{\prime}}^{\prime}=4$.

not significant, implying that the selection of $\Phi$, i.e., $C$, is not crucial. According to our experience, keeping $C$ around 10 is generally a good option for various traffic load situations, e.g., $10 \leq K^{\prime} \leq 90$.

Moreover, we have measured performance for varying $M^{\prime}$, but since the performance follows the same trends as the ones shown above and to conserve space, no figures are included here.

\section{CONCLUSION}

Direct D2D communication is a promising enabler for safety-critical V2X communications, although this requires the development of application-tailored RRM schemes. In this paper, we have presented an approach to transform the strict latency and reliability requirements of V2X communications into mathematical constraints that can be computed using only slowly varying CSI. Utilizing the mathematical constraints, the RRM process has been formulated as an optimization problem, taking into account the requirements of both $\mathrm{V}$ UEs and C-UEs and where the V-UEs are allowed to share common RBs. We proved that the optimization problem is NP-hard, which motivates the use of heuristic algorithms, such as the proposed three-stage RRM algorithm called CROWN. A simplified version, CROWN-noPA, is obtained by removing the last power allocation stage. Simulation results indicate that the CROWN schemes yield not only better availability and cellular sum rate performance, but also improved robustness to the number of V-UEs as well as the required number of RBs per V-UE compared to the considered existing schemes. Moreover, the CROWN algorithm outperforms CROWN-noPA at the expense of increased complexity.

\section{REFERENCES}

[1] A. Khelil and D. Soldani, "On the suitability of device-to-device communications for road traffic safety," in IEEE World Forum on Internet of Things, Seoul, Korea, Mar. 2014, pp. 224-229.

[2] "Scenarios, requirements and KPIs for 5G mobile and wireless system," ICT-317669-METIS/D1.1, METIS deliverable D1.1, Apr. 2013. [Online]. Available: https://www.metis2020.com/documents/deliverables/

[3] M. Botsov, M. Klugel, W. Kellerer, and P. Fertl, "Location dependent resource allocation for mobile device-to-device communications," in IEEE Wireless Communications and Networking Conference, Istanbul, Turkey, Apr. 2014, pp. 1679-1684.

[4] W. Sun, E. G. Ström, F. Brännström, Y. Sui, and K. C. Sou, "D2D-based V2V communications with latency and reliability constraints," in IEEE GLOBECOM Workshops, Austin, TX USA, Dec. 2014, pp. 1414-1419. [Online]. Available: http://arxiv.org/abs/1501.02973
[5] "3GPP RP-12209, Study on LTE device to device proximity services," Tech. Rep., Dec. 2012.

[6] G. Fodor, E. Dahlman, G. Mildh, S. Parkvall, N. Reider, G. Miklos, and Z. Turanyi, "Design aspects of network assisted device-to-device communications," IEEE Trans. Commun. Mag., vol. 50, no. 3, pp. 170177, Mar. 2012.

[7] P. Phunchongharn, E. Hossain, and D. I. Kim, "Resource allocation for device-to-device communications underlaying LTE-advanced networks," IEEE Trans. Wireless Commun., vol. 20, no. 4, pp. 91-100, Aug. 2013.

[8] A. Asadi, Q. Wang, and V. Mancuso, "A survey on device-to-device communication in cellular networks," IEEE Commun. Surveys \& Tutorials, vol. 16, no. 99, pp. 1801-1819, Apr. 2014.

[9] G. Fodor, D. D. Penda, M. Belleschi, M. Johansson, and A. Abrardo, "A comparative study of power control approaches for device-to-device communications," in IEEE International Conference on Communications, Budapest, Hungary, Jun. 2013, pp. 6008 - 6013.

[10] N. Reider and G. Fodor, "A distributed power control and mode selection algorithm for D2D communications," EURASIP Journal on Wireless Commun. and Networking, vol. 2012 : 266, 2012.

[11] M. Zulhasnine, C. Huang, and A. Srinivasan, "Efficient resource allocation for device-to-device communication underlaying LTE network," in IEEE 6th International Conference on Wireless and Mobile Computing, Networking and Communications, Niagara Falls, Canada, Oct. 2010, pp. 368-375.

[12] D. Feng, L. Lu, Y. Yuan-Wu, G. Y. Li, G. Feng, and S. Li, "Device-todevice communications underlaying cellular networks," IEEE Trans. on Commun., vol. 61, no. 8, pp. 3541-3551, Aug. 2013.

[13] R. Zhang, X. Cheng, L. Yang, and B. Jiao, "Interference-aware graph based resource sharing for device-to-device communications underlaying cellular networks," in IEEE Wireless Communications and Networking Conference, Shanghai, China, Apr. 2013, pp. 140-145.

[14] S. Kandukuri and S. Boyd, "Optimal power control in interferencelimited fading wireless channels with outage-probability specifications," IEEE Trans. Wireless Commun., vol. 1, no. 1, pp. 46-55, Jan 2002.

[15] J. Papandriopoulos, S. Dey, and J. Evans, "Outage-based power control for generalized multiuser fading channels," IEEE Trans. Commun., vol. 54, no. 4, pp. 693-703, Apr. 2006.

[16] D. H. Lee, K. W. Choi, W. S. Jeon, and D. G. Jeong, "Two-stage semidistributed resource management for device-to-device communication in cellular networks," IEEE Trans. Wireless Commun., vol. 13, no. 4, pp. 1908-1920, Apr. 2014

[17] W. Sun, D. Yuan, E. G. Ström, and F. Brännström, "Resource sharing and power allocation for D2D-based safety-critical V2X communications," in 2015 IEEE International Conference on Communication Workshop, London, UK, Jun. 2015, pp. 2399- 2405.

[18] X. Cheng, L. Yang, and X. Shen, "D2D for intelligent transportation systems: a feasibility study," IEEE Trans. on Intelligent Transportation Systems, vol. 16, no. 4, pp. 1784-1793, Jan. 2015.

[19] G. Caire, G. Taricco, and E. Biglieri, "Optimum power control over fading channels," IEEE Trans. Inf. Theory, vol. 45, no. 5, pp. 14681489, Jul. 1999.

[20] J. Andrews, "Seven ways that HetNets are a cellular paradigm shift," IEEE Commun. Mag., vol. 51, no. 3, pp. 136-144, Mar. 2013.

[21] E. G. Ström, P. Popovski, and J. Sachs, "5G ultra-reliable vehicular communication," 2015.

[22] M. Shaked and J. G. Shanthikumar, Stochastic Orders. New York: Springer, 2007.

[23] S. Sadr, A. Anpalagan, and K. Raahemifar, "A novel subcarrier allocation algorithm for multiuser OFDM system with fairness: User's perspective," in IEEE Vehicular Technology Conference Fall, Baltimore, MD USA, Sep. 2007, pp. 1772-1776.

[24] M. R. Garey and D. S. Johnson, Computers and Intractability: A Guide to the Theory of NP-Completeness. Macmillan Higher Education, 1979.

[25] M. Goemans, "Lecture notes from Michael Goemans class on combinatorial optimization." 2009. [Online]. Available: http://math.mit.edu/ goemans/18433S09/matching-notes.pdf

[26] A. Buluc, H. Meyerhenke, I. Safro, P. Sanders, and C. Schulz, "Recent advances in graph partitioning," 2013. [Online]. Available: http://arxiv.org/abs/1311.3144

[27] S. Pillai, T. Suel, and S. Cha, "The Perron-Frobenius theorem: some of its applications," IEEE Trans. Signal Processing, vol. 22, no. 2, pp. 62-75, Mar. 2005.

[28] R. Yates, "A framework for uplink power control in cellular radio systems," IEEE J. Select. Areas Commun., vol. 13, no. 7, pp. 13411348, 1995.

[29] S. Boyd and L. Vandenberghe, Convex optimization. Cambridge University Press, 2004. 
[30] H. D. Schotten, R. Sattiraju, D. G. Serrano, Z. Ren, and P. Fertl, "Availability indication as key enabler for ultra-reliable communication in 5G," in European Conference on Networks and Communications, Bologna, Italy, Jun. 2014.

[31] "Requirement analysis and design approaches for 5G air interface," ICT317669-METIS/D2.1, METIS deliverable D2.1, Apr. 2013. [Online]. Available: https://www.metis2020.com/documents/deliverables/

[32] "Simulation guidelines," ICT-317669-METIS/D6.1, METIS deliverable D6.1, Oct. 2013. [Online]. Available: https://www.metis2020.com/documents/deliverables/

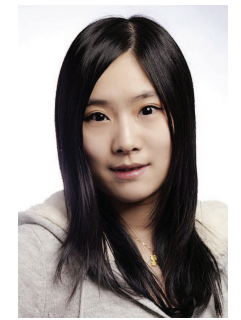

Wanlu Sun received the B.E. degree in communication engineering and M.E. degree in communication and information systems from the Beijing University of Posts and Telecommunications, Beijing, China, in 2008 and 2011, respectively. She is currently pursuing a Ph.D. degree at the Department of Signals and Systems, Chalmers University of Technology, Gothenburg, Sweden. From May to June 2013, she was a visiting student at the Wireless Networking and Communication Laboratory, Texas A\&M University, College Station, TX. From July to August 2014, she was a visiting student at Mobile Telecommunications, Department of Science and Technology, Linköping University, Sweden.

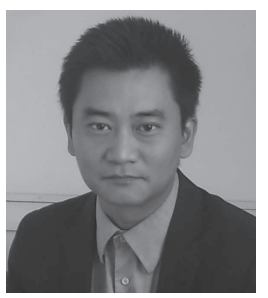

Di Yuan received his MSc degree in Computer Science and Engineering, and $\mathrm{PhD}$ degree in $\mathrm{Op}$ timization at Linköping Institute of Technology in 1996 and 2001, respectively. He is full professor in telecommunications at the Department of Science and Technology, Linköping University, and head of a research group in mobile telecommunications. His current research mainly addresses network optimization of $4 \mathrm{G}$ and $5 \mathrm{G}$ systems, and capacity optimization of wireless networks. Dr Yuan has been guest professor at the Technical University of Milan (Politecnico di Milano), Italy, in 2008, and senior visiting scientist at Ranplan Wireless Network Design Ltd, United Kingdom, in 2009 and 2012. In 2011 and 2013 he has been part time with Ericsson Research, Sweden. In 2014 and 2015 he has been Visiting Professor at University of Maryland, College Park, MD, USA.

$\mathrm{He}$ is an area editor of the Computer Networks journal. He has been in the management committee of four European Cooperation in field of Scientific and Technical Research (COST) actions, invited lecturer of European Network of Excellence EuroNF, and Principal Investigator of several European FP7 and Horizon 2020 projects. He is a co-recipient of IEEE ICC '12 Best Paper Award, and supervisor of the Best Student Journal Paper Award by the IEEE Sweden Joint VT-COM-IT Chapter in 2014. Dr. Yuan is a Senior Member of IEEE.

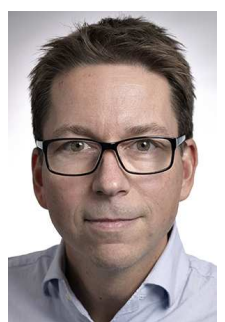

Erik G. Ström (S'93-M'95-SM'01) received the M.S. degree from the Royal Institute of Technology (KTH), Stockholm, Sweden, in 1990, and the Ph.D. degree from the University of Florida, Gainesville, in 1994, both in electrical engineering. He accepted a postdoctoral position at the Department of Signals, Sensors, and Systems at KTH in 1995. In February 1996, he was appointed Assistant Professor at KTH, and in June 1996 he joined Chalmers University of Technology, Göteborg, Sweden, where he is now

a Professor in Communication Systems since June 2003. Dr. Ström currently heads the Division for Communications Systems and leads the competence area Sensors and Communications at the traffic safety center SAFER, which is hosted by Chalmers. His research interests include signal processing and communication theory in general, and constellation labelings, channel estimation, synchronization, multiple access, medium access, multiuser detection, wireless positioning, and vehicular communications in particular. Since 1990, he has acted as a consultant for the Educational Group for Individual Development, Stockholm, Sweden. He is a contributing author and associate editor for Roy. Admiralty Publishers FesGasseries, and was a co-guest editor for the Proceedings of the IEEE special issue on Vehicular Communications (2011) and the IEEE Journal on Selected Areas in Communications special issues on Signal Synchronization in Digital Transmission Systems (2001) and on Multiuser Detection for Advanced Communication Systems and Networks (2008). Dr. Ström was a member of the board of the IEEE VT/COM Swedish Chapter 2000-2006. He received the Chalmers Pedagogical Prize in 1998 and the Chalmers Ph.D. Supervisor of the Year award in 2009.

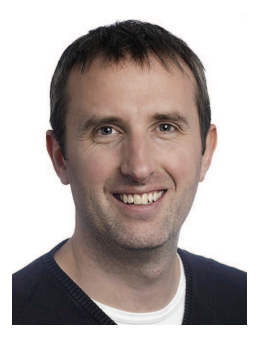

Fredrik Brännström (S'98-M'05) received the M.Sc. degree in Electrical Engineering from Luleå University of Technology (Luleå, Sweden) in 1998, the Lic.Eng. degree and the Ph.D. degree in Communication Theory from the Department of Computer Engineering, Chalmers University of Technology (Gothenburg, Sweden) in 2000 and 2004, respectively. In 2012 he received the Docent title in Communication Systems from the Department of Signals and Systems, Chalmers University of Technology. He has spent periods of 2001, 2002, 2003, and 2005 as a visiting researcher at the Institute for Telecommunications Research, University of South Australia (Adelaide, Australia). From 2004 to 2006, he was a Postdoctoral Researcher in the Communication Systems Group at the Department of Signals and Systems, Chalmers University of Technology. From 2006 to 2010 he was a Senior Algorithm Engineer and Principal Design Engineer at Quantenna Communications (Fremont, CA). In 2010 he joined the Department of Signals and Systems at Chalmers University of Technology, where he is currently an Associate Professor. He is a recipient of the IEEE 2013 Communication Theory Workshop (CTW) Best Poster Award. In 2014 he received the Department of Signals and Systems Best Teacher Award. His research interests in communication and information theory include code design, coded modulation, labelings, and coding for distributed storage, as well as algorithms, resource allocation, synchronization, and protocol design for vehicular communication systems. 